\title{
Assessment of breakup severity on operational satellites
}

\author{
Francesca Letizia* \\ Astronautics Research Group, University of Southampton, Southampton, SO17 1BJ, UK \\ Camilla Colombo \\ Astronautics Research Group, University of Southampton, Southampton, SO17 1BJ, UK, \\ c.colombo@soton.ac.uk \\ Hugh G. Lewis \\ Astronautics Research Group, University of Southampton, Southampton, SO17 1BJ, UK, \\ h.g.lewis@ac.uk \\ Holger Krag \\ ESA Space Debris Office, ESOC,64293 Darmstadt, Germany, Holger.Krag@esa.int
}

\begin{abstract}
In the past years, several methods have been proposed to rank spacecraft and space debris objects depending on their effect on the space environment. The interest in this kind of indices is primarily motivated by the need of prioritising potential candidates of active debris removal missions and to decide on the required reliability for disposal actions during the design phase. The index proposed in this work measures the effect of the catastrophic fragmentation of the analysed spacecraft in terms of the resulting collision probability for operational spacecraft. The propagation of the debris cloud generated by the fragmentation and the estimation of the collision probability are obtained by applying an analytical approach based on the study of the density of the fragment cloud. The dependence of the proposed severity index on the mass of the spacecraft and on its semi-major axis and inclination is investigated. The index was computed for the objects in the DISCOS database and its results were compared to
\end{abstract}

\footnotetext{
* Corresponding author

Email address: f.letizia@soton.ac.uk (Francesca Letizia)
}

Preprint submitted to Advances in Space Research

May 13, 2016 
other formulations proposed in literature. A discussion on the results and on the comparison is presented.

Keywords: space debris, environmental index, debris cloud

\section{Introduction}

The long term evolution of the space debris environment appears to be highly affected by fragmentations of massive objects, such as intact large spacecraft and rocket bodies (Rossi et al. 2015b). For this reason, different metrics have been proposed to rank spacecraft depending on the consequences of their fragmentation on the space environment. The purpose of these analyses is twofold. First, one objective is to obtain a better insight on the critical parameters that have the largest influence on the space debris evolution. Second, the output of these rankings could lead to the identification of potential candidates for active debris removal missions: in such a scenario, it would be important to decide which spacecraft should be removed first to have the largest global beneficial effect.

Several authors have proposed different approaches to the problem and highlighted the relevance of having a quantitative measure of the environmental effect of an object in orbit, depending on its orbital parameters and physical characteristics (Utzmann et al., 2012, Bastida Virgili and Krag, 2013, Lang et al., 2013, Rossi et al., 2015b). Rossi et al. (2015a), for example, simulated different fragmentations, considering locations and targets representative of the distribution of intact objects in orbit. For each scenario, the number of objects present in orbit in the 200 years following the fragmentation was studied and used to measure the effect of the fragmentation. Alternatively, Rossi et al. (2015b) introduced a criticality index, which depends on the background debris density, the object residual lifetime, the mass, and its orbital inclination. Similar parameters were identified also by Utzmann et al. (2012). In these case, no simulation is performed, and the indices collect what are identified as the most relevant factors to provide an immediate measure of the criticality of the studied object. A different approach was presented by Lewis (2014), where the 
proposed environmental index is computed considering the spacecraft orbital region, the implementation of mitigation measures for the spacecraft and the long term effect of the selected measure.

These examples show how the proposed environmental indices focus on different aspects of the space debris environment, ranging from the likelihood of the breakup to happen to the evaluation of the long-term changes in the whole debris population. In the ECOB index (Environmental Consequences of Orbital Breakups) proposed in this work, only the effects of potential breakups, of spacecraft and rocket bodies, are studied. For this reason, ECOB is indicated in the following also as a severity index to stress that it does not consider the likelihood of breakups to happen, but only how dangerous they can be for other space objects. In particular, the effect of a breakup is measured by the resulting collision probability for a set of target spacecraft with the breakup fragments over time. A grid in semi-major axis, inclination, and mass is used to define possible initial conditions of the breakup. For each case, the evolution of the produced debris cloud is modelled applying an analytical method, which describes how the cloud density changes under the effect of atmospheric drag. Given the fragment density, the collision probability of the target is obtained applying the analogy with the kinetic theory of gases. Once the value of the index is know for any point in the grid, a simple interpolation can be used to compute the value of the index for any object.

The article is organised as follows. Section 2 will describe the debris cloud propagation method and Section 3 will introduce the structure of the proposed environmental index. Some preliminary results are presented in Section 4 and they will be used in Section 5 to specify the index computation. More detailed results will be presented in Section 6 and the comparison with other proposed environmental indices will be discussed in Section 7 . 


\section{Debris cloud propagation method}

According to the NASA breakup model (Krisko, 2011), for each trackable object produced by a fragmentation there are millions of objects in the size range between $1 \mathrm{~mm}$ and $5 \mathrm{~cm}$. Considering these numbers, even low intensity fragmentations can easily produce some thousands objects, whose individual propagation would make the simulation prohibitive in terms of computational resources (i.e. time and RAM). Evolutionary studies on the debris population usually deal with this issue by setting a cut-off fragment size at $10 \mathrm{~cm}$, so that only objects larger than this threshold are included in the simulations. However, especially when the impact of a single breakup is analysed, it could be relevant to include all objects that have the potential to interfere with other spacecraft, decreasing the threshold down to $1 \mathrm{~mm}$. This change in the scope of the analysis can be achieved by abandoning the evaluation of the single fragments' trajectories and studying the fragmentation cloud globally.

The propagation method CiELO (debris Cloud Evolution in Low Orbits) was developed with this aim: within this approach, the fragmentation cloud is described in terms of its spatial density, whose evolution in time under the effect of drag is obtained by applying the continuity equation. A detailed description of the method can be found in Letizia et al. (2015b), whereas only a brief overview of the approach is provided here, focussing mostly on the new improvements with respect to Letizia et al. (2015b).

The simulation of a fragmentation event starts with the modelling of the breakup. The NASA breakup model (Johnson and Krisko, 2001; Krisko, 2011) is used for this purpose. The evolution of the fragment cloud from this time instant is affected both by the dispersion of the energy among the fragments and the effect of orbit perturbations. Considering only the case of fragmentations in LEO, the Earth's oblateness spreads the fragments to form a band around the Earth. Once the band is formed, the atmospheric drag can be considered

as the main perturbation and the continuity equation can be applied to obtain the cloud density evolution, following the approach firstly proposed by McInnes 
(1993).

Compared to formulation by McInnes (1993), where the debris density is function of the radial distance from the Earth $(r)$ only, the method was extended to express the cloud density as function of semi-major axis $(a)$ and eccentricity (e) (Letizia et al. 2015d). This extension results into an increase in the method applicability: whereas the description with the distance only can be applied to fragmentations starting from circular orbits between 800 and $1000 \mathrm{~km}$, the formulation in $a$ and $e$ can be used also for orbital altitudes between 700 and $800 \mathrm{~km}$. This means that the analytical method can be employed for the whole region where the majority of fragmentations occurred (Orbital Debris Program Office, 2014).

\subsection{Density-only formulation}

As briefly mentioned at the beginning of this section, the continuity equation is applied once the band is formed because only in that moment the hypotheses required to obtain an analytical solution hold. This means that alternative modelling techniques are required to describe the transition to the band. In Letizia et al. (2015b) this was done by numerically propagating the trajectory of the fragments for the months required to form the band. In the new version of the model used in this work this is done by applying a method similar to the one embedded in the continuity equation, which does not involve integrating the fragments' trajectories. When the continuity equation is solved with the method of characteristics, the value of the solution at a certain time is obtained by reshaping the initial condition according to the change prescribed by the conservation of the solution along the characteristics. Similarly, modelling the first phase of the cloud evolution is equivalent to describe how the $(a, e)$-plane changes from the initial time of breakup to the time of band formation $T_{B}$ to reproduce the evolution in Figure 1, which shows the distribution of fragments on the $(a, e)$-plane at the two time instants. Observe that this approach is equivalent to decompose the evolution of the cloud in two phases: a short-term one, driven by the Earth's oblateness, and a long-term one, driven by atmospheric 
0 days

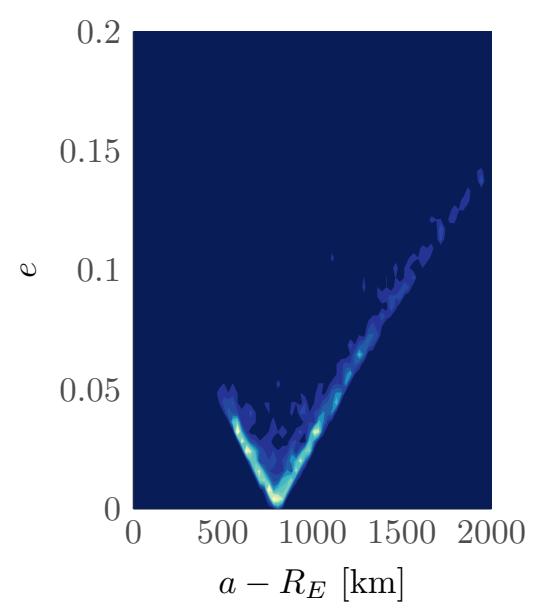

95.5 days

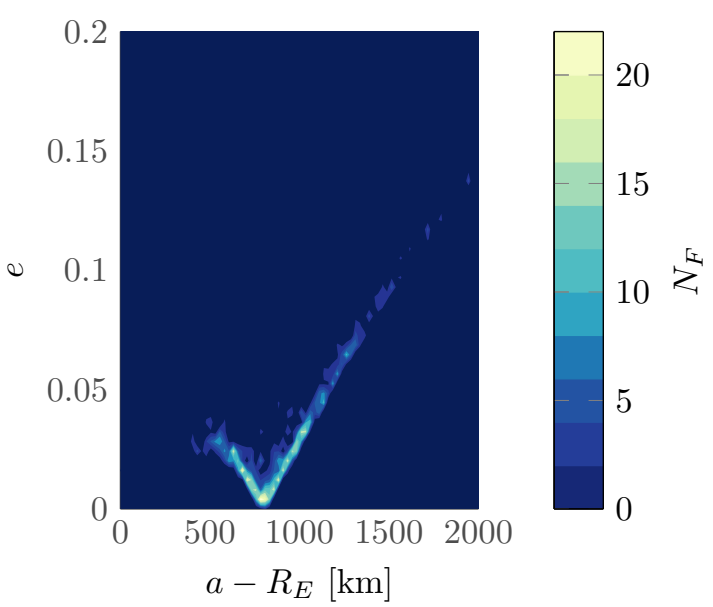

Figure 1: Visualisation of cloud density at the breakup and at the band formation $T_{B}$ for a fragmentation at $800 \mathrm{~km}$. $N_{F}$ indicates the number of fragments.

drag. As the Earth's oblateness does not affect the distribution in semi-major axis and eccentricity, the phase of spreading of the cloud to form a band is ignored. In other words, only the variation of the distribution in semi-major axis and eccentricity in the initial phase is modelled because it is the only element that affect the long term evolution of the cloud.

The method that describes the transition between the two states in Figure 1 is simplified by neglecting the variation of the eccentricity: only the variation of the semi-major axis a due to drag is considered. This is done purely for computational reasons, to reduce the number of reference trajectories that are considered. For each point in the $a$-axis it is possible to compute the variation of $a$ in the time $T_{B}$ and obtain a modified grid by applying the semi-analytical propagation method (Colombo, 2014) to evaluate the effect of drag. Observe that the variation in $a$ depends on the area-to-mass ratio, so the computation should be repeated for each $A / M$ bin in which the cloud is divided (Figure 2). It was shown that the optimal number is ten bins in $A / M$ (Letizia et al., 2015b). A similar approach was used by Rossi et al. (1998), who consider also the effect of eccentricity, to develop a fast propagator for debris clouds; in this case, 


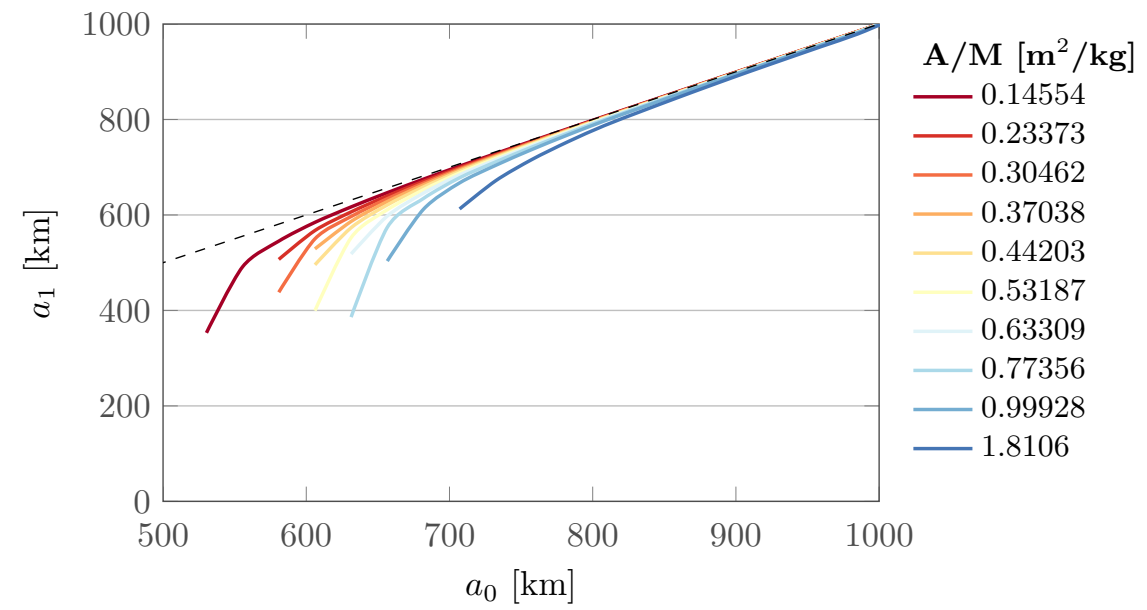

Figure 2: Resulting semi-major axis $a_{1}$ for the initial values of semi-major axis $a_{0}$ for all the $A / M$ bins for a propagation time equal to $T_{B}$.

this approach is used only for the initial evolution of the cloud, whereas the continuity equation is kept to describe the evolution when the band is formed.

Once each value of the initial grid is mapped into its modified value, it is possible to obtain the distribution $n\left(a, e, T_{B}\right)$ from the initial one $n\left(a, e, t_{0}\right)$ with the following algorithm. Let $a_{j}$ indicate the $j$-th point in the original grid and $a_{k}$ its modified value after $T_{B}$ : this value is compared with a threshold value $R_{E}+h_{\text {lim }}$, with $R_{E}$ radius of the Earth and $h_{\text {lim }}$ equal to $50 \mathrm{~km}$. If $a_{k}<R_{E}+h_{\text {lim }}$ the fragments originally at $a_{j}$ have re-entered and so $n\left(a, e, T_{B}\right)$ is not updated. If $a_{k}>R_{E}+h_{\lim }$, the corresponding value of the density when the band is formed $\left(n\left(a_{k}, e, T_{B}\right)\right)$ is obtained from the value at the breakup at the original semi-major axis $\left(n\left(a_{j}, e, t_{0}\right)\right)$, so $n\left(a_{k}, e, T_{B}\right)=n\left(a_{j}, e, t_{0}\right)$. In this way, the initial distribution $n_{0}(a, e)$ at the band formation is known. The long term propagation is obtained by applying the continuity equation as in the previous version of the method (Letizia et al., 2015d).

The density-only method was tested to verify that its results are coherent with the results obtained with the previous approach. Figure 3 compares the density profile obtained from the all analytical approach with the one of the 1D method described in Letizia et al. (2015b) (indicated as density for band 

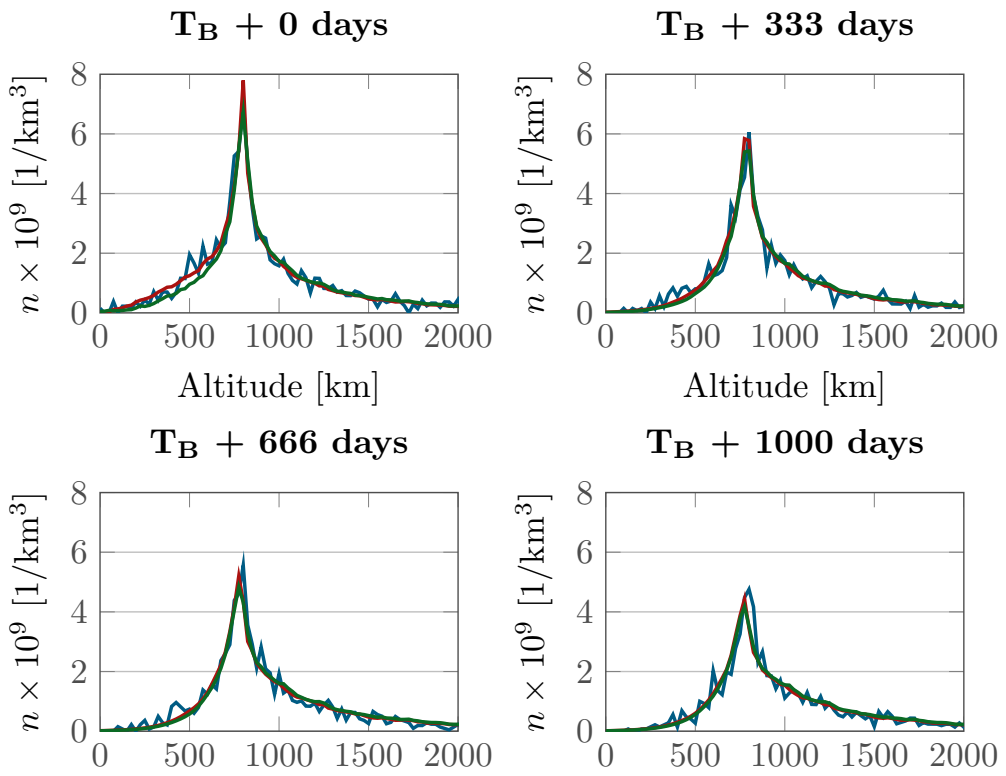

Altitude [km]

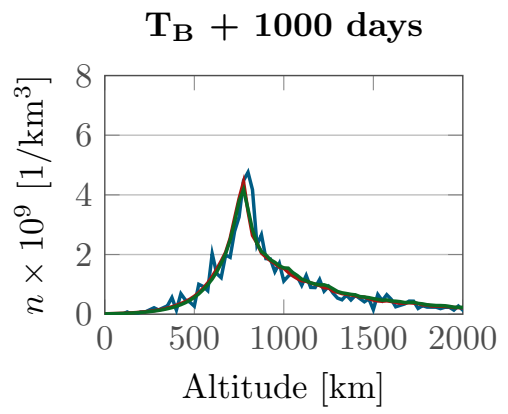

— Numerical
Density only (1D)

Figure 3: Fragment spatial density for a fragmentation at $800 \mathrm{~km}$. 
phase) and with the distribution obtained from the numerical propagation with a single run of the breakup model. In order to understand the applicability of density based approaches, only the effect of atmospheric drag is included in the numerical propagation as this is the only perturbation currently included in the continuum approach. Future work will aim to include additional perturbations in the density-based method, such as for example the solar radiation pressure, which are not included in the current study. The studied fragmentation is a non-catastrophic collision with a projectile of mass equal to $100 \mathrm{~g}$ and relative velocity equal to $1 \mathrm{~km} \mathrm{~s}^{-1}$ and results in 2397 fragments at the breakup. The two methods (density for band phase and density-only) differ only for how the initial condition (at $T_{B}$ ) is obtained, whereas the long term propagation is obtained with the same method. One can observe how the density profile of the density-only method is very similar to the one where the density is used only in the band phase. In particular, the difference between the integral of the density profile obtained with an analytical method $\left(n_{A}\right)$ and the one from numerical propagation $\left(n_{N}\right)$ is lower than $10 \%$ for both cases (density for band phase and density-only). Another metric of the accuracy of the method is defined as

$$
\operatorname{err}_{\text {prof }}=\frac{\int\left|n_{A}-n_{N}\right| d h}{\int n_{N} d h}
$$

which measures the mean absolute error between two density profiles. In both cases, $\operatorname{err}_{\text {prof }}=0.29$ was found, in line with previous results (Letizia, 2016). Same results were obtained for other values of inclinations (i.e. $30^{\circ}$ and $60^{\circ}$ ) suggesting that, as observed with the density for band phase approach, the accuracy does not depend on the inclination. These results confirm that the short term evolution of the trajectory of the fragments can be neglected if one is interested only in the evolution of the spatial density in the long term.

This variation in the model is particularly important because removing completely the numerical propagation of the fragments' trajectory, also for a short phase as in (Letizia et al. 2015b), makes the computational effort of the model really independent on the number of fragments contained in the cloud. In this 


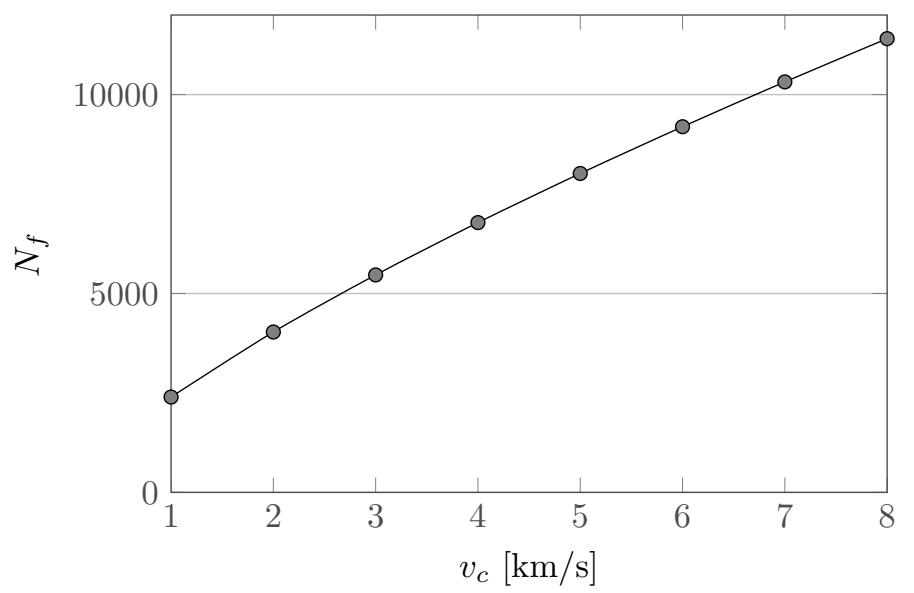

Figure 4: Number of fragments produced by a non-catastrophic collision with a projectile of $100 \mathrm{~g}$ as a function of the impact velocity $v_{c}$ according to NASA breakup model (Johnson and Krisko, 2001).

way, any breakup can be simulated with the same computational time. In addition, also large events can be simulated without supercomputer facilities as also the request of RAM is fixed. This was verified by studying fragmentations with increasing number of objects.

\subsection{Applicability to large clouds}

In the case of non-catastrophic collisions, the number of fragments produced by a breakup depends on the collision velocity $v_{c}$ (Johnson and Krisko, 2001). Figure 4 shows the dependence on the number of the produced fragments on the collision velocity for a non-catastrophic collision with a projectile of $100 \mathrm{~g}$. A way to test the density-only approach is to simulate breakups with increasing collision velocity to evaluate its computational time and assess whether, adopting this formulation, its dependence on the number of fragments is removed. Figure 5 shows the computational time for the simulation of breakup clouds resulting from non-catastrophic collisions with $v_{c}$ between 1 and $8 \mathrm{~km} / \mathrm{s}$ and total fragment number ranging between 2000 and 12000 . In all cases, the computational time was always around $11 \mathrm{~s}$ on a machine with $4 \mathrm{CPU}$ and $16 \mathrm{~GB}$ of RAM. There is only a weak dependence of the computational time on the num- 


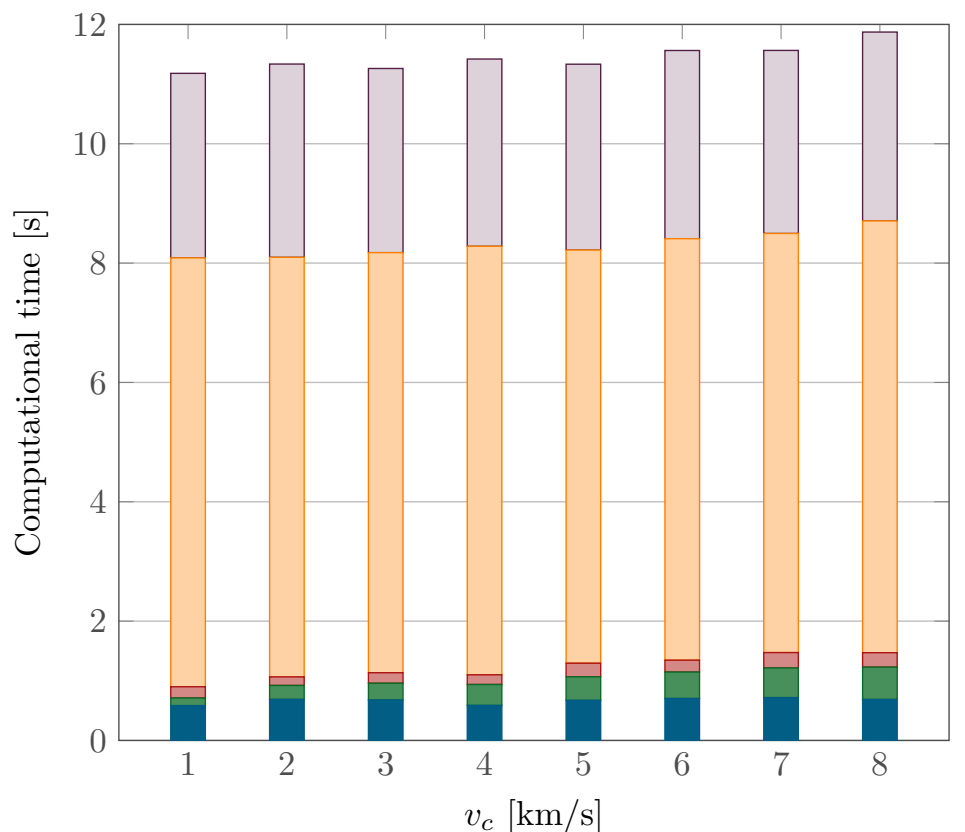

Breakup
$\square n_{0}$ reshape $\quad \square$ iv analyion in bin $\quad \square$ Initial condition

Figure 5: Computational time with the density-only method for non-catastrophic collisions with projectile mass equal to $100 \mathrm{~g}$ and different collision velocity $v_{c}$. 
ber of fragments and this is due to the process of grouping the fragments into the $A / M$ bins; once this phase is complete, the problem is formulated purely in spatial density and its propagation is independent from the number of fragments. This aspect makes the proposed density-only method very promising for evaluating many collision and explosion scenarios with different energies.

\subsection{Continuity equation}

Once $n_{0}$ is known, the continuity equation can be used to obtain the long term evolution of the density $n$. The evolution of the fragment density in the space of orbital parameters is written as (Letizia et al., 2015d)

$$
n(a, e, t)=n_{0}(a, e) \frac{v_{a}\left(a_{i}\right)}{v_{a}(a)}
$$

where $v_{a}$ is the rate of variation of the semi-major axis due to atmospheric

drag. The expression of $v_{a}$ is derived from King-Hele (1987) and simplified in the following expression to solve the problem analytically

$$
v_{a}(a)=-\sqrt{\mu_{E} R_{F}} \frac{c_{D} A}{M} \rho_{0} \exp \left(-\frac{a-R_{F}}{H}\right) f\left(R_{F}, \tilde{e}(a), H\right),
$$

with $\mu_{E}$ gravitational constant of the Earth, $R_{F}$ radial distance where the fragmentation occurred, $c_{D}$ and $A / M$ respectively drag coefficient and area-to-mass ratio of the fragments, $\rho_{0}$ and $H$ atmospheric density and scale height at $R_{F}$. $f\left(R_{F}, \tilde{e}(a), H\right)$ is a function that collects the Bessel functions, which describe the effect of eccentricity

$$
f=I_{0}(z)+2 e I_{1}(z)+O\left(e^{2}\right)
$$

where $I_{j}$ is the Bessel function of the first kind and order $j$ with argument $z=$ $R_{F} \tilde{e}(a) / H$. The function $\tilde{e}(a)$ describes the initial distribution of eccentricity with semi-major axis. This means that the cloud is propagated with the (strong) assumption that the distribution of eccentricity with semi-major axis is constant through the whole simulation. Further details can be retrieved in (Letizia et al. 
2015d).

The value of the cloud spatial density is then obtained from the density in the space of orbital elements by applying, to the whole domain in $(a, e)$, expressions such as the ones by Kessler (1981) and Sykes (1990) to compute the probability of finding an object given its orbital parameters. The expression used is

$$
s(r)=\frac{1}{4 \pi r a^{2}} \frac{1}{\sqrt{e^{2}-\left(\frac{r}{a}-1\right)^{2}}},
$$

where $s(r)$ indicates the density as a function of the radial distance.

It is important to highlight that the continuity equation is used to model only the density as a function of the geocentric distance, whereas the cloud density depends also on the latitude and this should be taken into account when assessing the collision probability for a spacecraft crossing the fragment cloud. Among others, Kessler (1981) and Sykes (1990) have shown that the dependence on the distance $r$ and on the latitude $\beta$ can be described separately expressing the density $S$ as the product of two components:

$$
S(r, \beta)=s(r) f(\beta) .
$$

This is particularly useful in the application to debris clouds as the evolution of the two parameters occurs with different time scales and drivers. In fact, the purpose of the proposed method is to study the long term (i.e. years) effect of a fragmentation, whereas the latitude of a target spacecraft crossing the cloud evolves in a much shorter time scale (i.e. hours). Following the target latitude would require very short time steps for the integration, eliminating or reducing the advantage of having a fast propagator for the fragment cloud. For this reason, an average value over $\beta$ is considered. The average density value can be found computing once the integral average of $f(\beta)$ over one orbit period and applying it to rescale the spatial density at any time, recalling the hypothesis that the fragments' and the target's inclinations are not changing as the rotation of the Earth's atmosphere is neglected. The dependence of the latitude $\beta$ on 
the orbital parameters is expressed by

$$
\beta=\arcsin [\sin (\omega+\nu) \sin i]
$$

where $\omega, \nu, i$ refer, respectively, to the argument of perigee, the true anomaly and the inclination of the target spacecraft crossing the cloud. Introducing the argument of latitude $u=\omega+\nu$ and writing the expression for the case of a target on a circular orbit, the scale factor of the spatial density can be computed as

$$
\bar{f}=\frac{1}{2 \beta_{\max }} \int_{0}^{2 \pi} \frac{\mathrm{d} u}{\sqrt{\cos ^{2}(\beta(u))-\cos ^{2}\left(\beta_{\max }\right)}}
$$

where $\beta(u)$ is given by Equation 7, $\beta_{\max }$ is the maximum latitude covered by

the band. For non-equatorial orbits $\beta_{\max }$ is set equal to the inclination where the fragmentation occurred $i_{F}$ if $i_{F} \leq \pi / 2$ and equal to $\pi-i_{f}$ otherwise (Letizia et al., 2015c).

\subsection{Collision probability}

Once the cloud density at any time is known, it is possible to evaluate its effect on the collision probability for a spacecraft that crosses the cloud. The computation of the collision probability is based on the average number of collisions $N$ in an interval of time (Kessler, 1981). This number is then used to obtain the cumulative collision probability for the target spacecraft through a Poisson distribution

$$
p_{c}(t)=1-\exp (-N)
$$

following the commonly used analogy with the kinetic gas theory (McKnight, 1990: Su and Kessler, 1985). Note that this analogy can be applied when the motion of different fragments is not correlated (Jenkin, 1996). The average number of collisions $N$ in a given interval of time $\Delta t=t-t_{0}$ can be written as

$$
N=F \sigma \Delta t
$$


where $F$ is the flux of particles and $\sigma$ represents the collisional cross-sectional area Kessler (1981). This last parameter is usually defined considering the dimensions of both the colliding objects Kessler (1981), but, in this work, it is substituted by the cross-sectional area of the target spacecraft. This is due to the fact that the only to objects smaller than $10 \mathrm{~cm}$ are considered in the current analysis as the application to a continuum approach to larger objects could be questionable. For this reason, the fragments in the cloud are much smaller than target satellite, so $\sigma \approx A_{c}$. The flux $F$ is equal to

$$
F=S(r, t) \Delta v
$$

where $S(r, t)$ is the value of the spatial density obtained with the analytical method based on the continuity equation and applying the scaling factor due to the distribution in latitude in Equation $8, \Delta v$ is the average relative velocity between the targets and the fragments, which is also obtained from the orbital configuration of the target and the fragmentation (Letizia et al. 2015a). In details,

$$
\Delta v=\frac{\iint \mathbf{C}(a, e) \mathbf{n}(a, e) \delta \mathbf{v}(a, e) d a d e}{\iint \mathbf{C}(a, e) \mathbf{n}(a, e) d a d e}
$$

with

$$
\mathbf{C}\left(a_{j}, e_{k}\right)=1 \quad \text { if } a_{j}\left(1-e_{k}\right) \leq r_{T} \leq a_{j}\left(1+e_{k}\right)
$$

and $\mathbf{C}\left(a_{j}, e_{k}\right)=0$ otherwise. In Equation 12, $\delta v$ indicates the estimated relative velocity between the target and the points in the $(a, e)$-plane where $n$ is evaluated

$$
\delta v=\frac{2}{\pi} \sqrt{\chi+\eta} \mathrm{E}\left[\frac{2 \eta}{\chi+\eta}\right]
$$

with E complete elliptic integral of the second kind and

$$
\begin{aligned}
& \chi=v_{T}^{2}+v_{F}^{2}-2 v_{T} v_{F} \cos \gamma_{F} \cos i_{T} \cos i_{F} \\
& \eta=2 v_{T} v_{F} \cos \gamma_{F} \sin i_{T} \sin i_{F},
\end{aligned}
$$


where

$$
v_{F}=\sqrt{2 \mu\left(\frac{1}{r_{T}}-\frac{1}{2 a_{F}}\right)} \quad \cos ^{2} \gamma_{F}=\frac{a_{F}\left(1-e_{F}^{2}\right)}{r_{T}\left(2 a_{F}-1\right)} ;
$$

the subscripts $F$ and $T$ refers to quantity of the fragments and of the target respectively.

\section{Environmental index}

The ability of the density-only formulation to model large debris clouds with limited computational effort (both in terms of simulation time and RAM) makes it suitable to simulate a large number of breakup scenarios and build an environmental index based on the assessment of their consequences. In this work, the breakups of specific space objects are evaluated in terms of the effect on a set of target objects, which represent the active satellites in LEO. This is done to assess how a breakup affects the operational aspects of space debris activities, namely if an increase in close conjunction alerts and collision avoidance manoeuvres should be expected. In addition, using active satellites as reference targets for the environmental index means that the consequences of a fragmentation are related to a potential loss for the spacecraft operators. An environmental index could also be used, prior to launch, to support the licensing phase in the evaluation of the planned post-mission disposal strategies. For example, a licence system connected to an environmental index can distinguish between a CubeSat in orbit at $700 \mathrm{~km}$ at low inclination and a $4000 \mathrm{~kg}$ satellite in a polar orbit at $800 \mathrm{~km}$. As the environmental impact of a potential breakup involving the two spacecraft is different, a higher level of reliability of the post-mission disposal may be requested in the latter case.

The propagation method CiELO, with the density-only approach described in Section 2, allows the space of the most relevant object parameters (i.e. altitude, orbital inclination, and mass) to be mapped onto a value of environmental index, enabling to identify the most critical orbital configurations. This approach requires two steps. First, the potential sources of fragmentations and which kind of fragmentations to simulate should be defined. Second, the target set should 
be represented in such a way that the propagation of all the active satellites' trajectories is not required. The next Sections will explain how these tasks are performed. Observe that the structure of the index can be applied also with different cloud propagation methods than CiELO, given that they can provide the cloud spatial density with time. As an example, the same index structure could be applied in GEO (Geosynchronous Earth Orbit), where different analytical propagation methods are available (Valk et al. 2009), to study fragmentations within the GEO protected region and in the GEO graveyard orbit.

\subsection{Sources of fragmentation}

To keep the severity index as general as possible, a set of fictitious sources is defined to map the space of parameters for any possible space object; in other words, a large set of virtual fragmentations is created. In particular, a grid in semi-major axis, inclination, and spacecraft mass was used. The extremes of the grid in semi-major axis are limited by the applicability of the method: the highest limit is set equal to $1000 \mathrm{~km}$ as for higher altitudes it will not be justifiable to model the atmospheric drag and not the solar radiation pressure. The lowest limit depends on the analytical formulation used for the propagation, which is applicable to fragmentation above $700 \mathrm{~km}$ for a propagation period around 25 years (Letizia et al., 2015d). For the mass of the fragmentation source, a grid between $100 \mathrm{~kg}$ and $10000 \mathrm{~kg}$ was defined. Observe that the only way to consider the effect of the fragmenting mass within the NASA breakup model requires simulating catastrophic collisions and not explosions or non-catastrophic collisions. For all the simulated collisions the breakup of a spacecraft (rather than a rocket body) is assumed, even if in the size range of interest the impact of this hypothesis is minima 1 . The collision velocity is set equal to $10 \mathrm{~km} \mathrm{~s}^{-1}$, which is an average value for LEO and which was used also by Rossi et al. (2015a). The mass of the colliding projectile is neglected. Using the NASA breakup model,

\footnotetext{
${ }^{1}$ The kind of object involved in the fragmentation affects the definition of the parameters of fragments larger than $8 \mathrm{~cm}$ only.
} 
the simulation of a catastrophic collision involving a mass equal to $1000 \mathrm{~kg}$ results in the generation of 2.4 million fragments larger than $1 \mathrm{~mm}$. Even if the density-only formulation is only weakly dependent on the number of fragments, this large value results in a long computational time only for the initialisation of the cloud. For this reason, the lowest cut-off size of the fragments was set equal to $1 \mathrm{~cm}$ for the results discussed in this work. With this threshold, the fragment cloud produced by the catastrophic collision of an object with mass equal to $1000 \mathrm{~kg}$ still contains more than 45000 objects with size between 1 and $10 \mathrm{~cm}$.

\subsection{Target set}

The effect of the virtual fragmentations is assessed on a set of spacecraft targets. The selection of the possible targets follows an approach similar to the one proposed by Rossi et al. (2015a) to define representative fragmentations. The cross-sectional area $A_{c}$ was identified as the most relevant parameter for a target. For this reason, the distribution of cross-sectional area across the LEO region was studied; also in this case, the semi-major axis was limited to $700 \mathrm{~km} \leq a \leq 1000 \mathrm{~km}$ as in the definition of the potential fragmentation sources. DISCOS database is used to extract the data of satellites orbiting in this region. Only satellites launched in the last ten years are included in the list, assuming that this criterion filters out inactive spacecraft. The studied LEO region is divided into cells in semi-major axis and inclination, applying the same grid used for the discretisation of possible fragmentation sources. For each cell, the cumulative cross-sectional area $\sum A_{c}$ is computed. An example of this analysis is shown in Figure 6 which represents the distribution of the cross-sectional area on a grid with spacing equal to $50 \mathrm{~km}$ in semi-major axis

and 30 degrees in inclination. The cells with the highest $\sum A_{c}$ indicate the the most vulnerable orbital regions in case of fragmentations.

Once the distribution of $A_{c}$ is known, the target set can be defined by selecting for each cell in Figure 6 a representative spacecraft for which the collision probability is computed. As it will be shown later, the environmental index is 


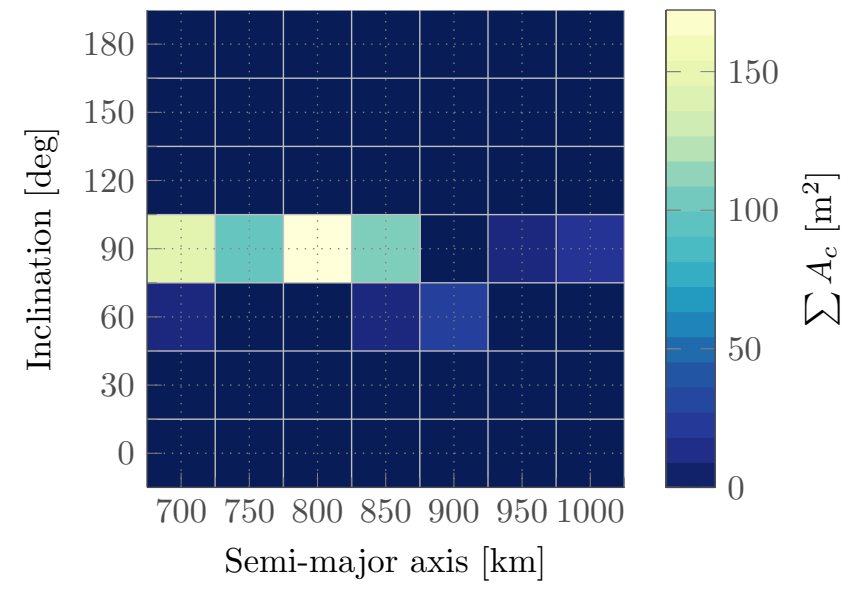

Figure 6: Distribution of cross-sectional area in the cells in semi-major axis and inclination. Data from DISCOS.

obtained by combining the collision probability for each target, weighting their contribution depending on the share of $A_{c}$ of the cell they represent. A fixed number of targets can be used or the code can select the appropriate number of targets to represent a given percentage of the total $A_{c}$. In the results shown in Section 6 the second approach is used, setting the percentage equal to $90 \%$ of the total $A_{c}$.

Two main options for the selection of targets exist. The first one identifies a real object among the ones in the cell, which is then used to represent the whole cell. The identification can be done, for example, by choosing the object with the maximum/minimum $A_{c}$ within the cell or the one with $A_{c}$ closest to the average value in the cell. The second option defines a synthetic object with $A_{c}$ and mass equal to the average values in the cell, semi-major axis and inclination equal to the centre of the cell.

Figure 7 and Figure 8 show the evolution of the semi-major axis of the representative object (coloured line) and of all the objects in the cell (in grey) for the two cells with the highest cumulative cross-sectional area. In Figure 7. the representative object is selected as the one with $A_{c}$ closest to average value in the cell, whereas in the second case, Figure 8, a synthetic target is defined. As 


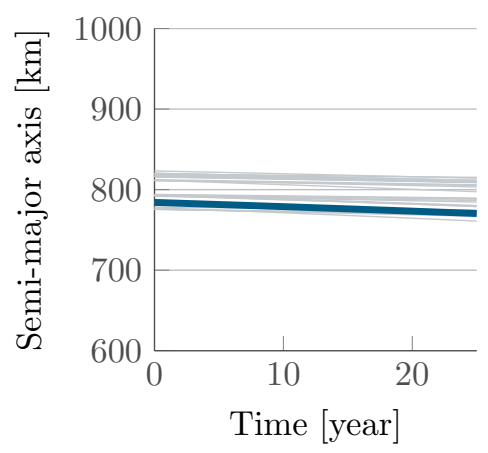

(a) Cell $1\left(90^{\circ}, 800 \mathrm{~km}\right)$

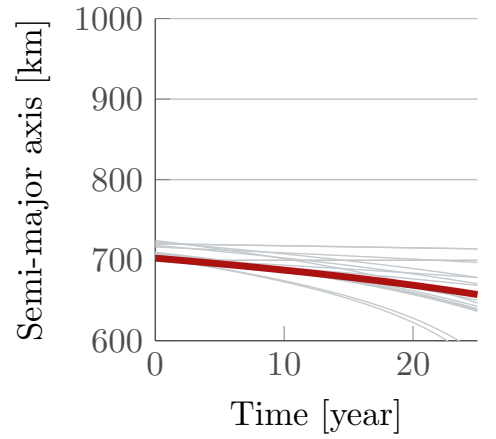

(b) Cell $2\left(90^{\circ}, 700 \mathrm{~km}\right)$

Figure 7: Evolution with time of the semi-major axis of the objects in a cell (in grey). The coloured line refer to the representative object ( $A_{c}$ closest to average value).

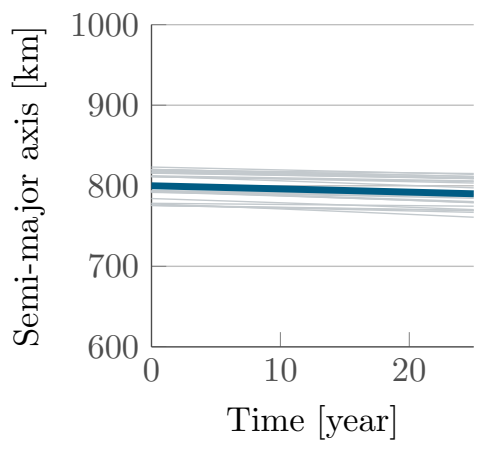

(a) Cell $1\left(90^{\circ}, 800 \mathrm{~km}\right)$

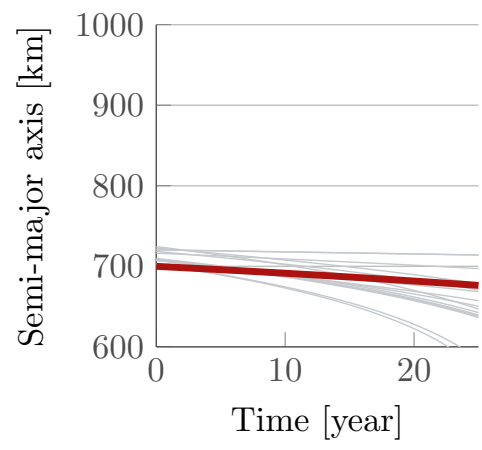

(b) Cell $2\left(90^{\circ}, 700 \mathrm{~km}\right)$

Figure 8: Evolution with time of the semi-major axis of the objects in a cell (in grey). The coloured line refer to the representative object (synthetic object). 
expected, a synthetic object has an evolution more representative of the whole cell. Moreover, choosing a synthetic object offers a more robust representation of the satellite population with time. In fact, the input population changes with time because of the launch of new satellites. This would require to re-run the algorithm for the selection of representative targets after each launch. In the current study, it is assumed that the development of space activities the development of space activities in LEO will be similar also in the future, with comparable missions to the current ones in each orbital regime. One could then expect a limited variation of the average cross-sectional area and mass. A tolerance on their value may be introduced to decide when the selection of the targets needs to be repeated. The other two options for the choice of the representative object, namely maximum and minimum $A_{c}$ within a cell, could be used only to define the worst/best case, but one has to consider that $A_{c}$ has a direct impact not only on the dynamics, through the effect of atmospheric drag, but also on the collision probability.

Figure 7 and Figure 8 were also used to assess if the propagation of the target trajectory is really required or if the orbit can be considered unperturbed. The object from cell $2\left(90^{\circ}, 700 \mathrm{~km}\right)$ is the one that experiences the maximum variation of the semi-major axis, equal to $40-50 \mathrm{~km}$, depending on the object definition; for the other cells the variation is lower than $15 \mathrm{~km}$. Given these numbers and the fact that the propagation of the targets' trajectories is not expensive in terms of computational time, it was decided to keep the effect of atmospheric drag and Earth's oblateness in the description of the targets' trajectories. An alternative approach could be not to propagate the targets assuming that if they are active satellites, their altitude may be controlled during mission lifetime. The evaluation of this option is left for future work.

\subsection{Index definition}

The purpose of the current analysis is to rank the sources of fragmentations evaluating their impact on a set of targets (i.e. the representative objects). As only the consequences of a breakup are evaluated, the proposed environmental 
index ECOB is defined as a simple sum of the collision probability on each target multiplied by the weighting factor $w_{j}$. When synthetic objects are used, the index is simply

$$
\mathrm{ECOB}=\sum_{j=1}^{N_{\text {tar }}} w_{j} p_{c, j}
$$

where $N_{\text {tar }}$ is the total number of representative objects; $p_{c, j}$ is the cumulative collision probability of the representative object of the $j$-th cell over the considered simulated time; $w_{j}$ is the weighting factor defined as

$$
w_{j}=\frac{\left(A_{c}\right)_{\mathrm{cell}, j}}{\left(A_{c}\right)_{\mathrm{tot}}},
$$

i.e. the ratio between the sum of $A_{c}$ in the $j$-th cell and the total $A_{c}$ on the whole target list. This definition of the weighting factor takes into account that each cell represents a different share of the total distribution of the crosssectional area. In this way, the targets associated to a cell with high $\left(A_{c}\right)_{\text {cell }}$ have a larger impact on the value of the index. When the representative object is chosen among one of the real satellites, Equation 15 may be modified in

$$
\mathrm{ECOB}=\sum_{j=1}^{N_{\mathrm{tar}}} k_{j} w_{j} p_{c, j}
$$

with

$$
k_{j}=\frac{\left(\bar{A}_{c}\right)_{\mathrm{cell}, j}}{\left(A_{c}\right)_{\mathrm{SC}, j}},
$$

to take into account the used value of $A_{c}$ is different from the average value in the cell.

Observe that the weighting factors $w_{j}$ are constant for the whole simulation, meaning that it is assumed that for the whole simulated time span the distribution of cross-sectional area will be constant. As mentioned before, this is equivalent to assume that, for the studied period, the space activities in the LEO region will be similar to the current ones. 


\section{Preliminary results}

Some preliminary runs were performed using six reference targets and a coarse grid with a step equal to $50 \mathrm{~km}$ for the semi-major axis and 30 degrees in inclination. The purpose of these runs is to analyse the general structure of the index.

\subsection{Effect of selection criteria}

In Section 3.2 different options for the selection of the targets were discussed. Figure 9 represents the value of ECOB computed on the coarse grid. It shows the comparison between the use of real spacecraft or synthetic objects to define the target set. When real spacecraft are used, the satellite with $A_{c}$ closest to the cell average is selected. The targets are superimposed to Figure 9 and indicated with the markers. The colour indicates the value of the index for a fragmenting spacecraft with values of semi-major axis and inclination equal to the ones of the centre of the cell. The mass of the virtual fragmenting spacecraft is equal to $1000 \mathrm{~kg}$. One can observe how the distribution of the index is qualitatively similar because the value of $A_{c}$ used in the two cases are close. This means that both the trajectory evolution and the computation of the collision probability will give similar results. This result can be interpreted also as a proof of the method robustness as the variation in semi-major axis and inclination within a single cell appear to have a limited impact on the final result.

\subsection{Effect of propagation time}

As introduced in Section 3, ECOB is function of the propagation time as it is obtained as the sum of the cumulative (over time) collision probability of the reference targets. The variation of the index with time was studied by producing a heat map similar to the ones in Figure 9 after different propagation periods. Figure 10 shows the results obtained after 5, 15, and 25 years. In this case, no weighting factor is applied to compose the index, so that the dependence on the time is more evident. In this way, one can observe that with longer time of propagation the relative importance of fragmentations at higher altitudes 


\section{5 years}

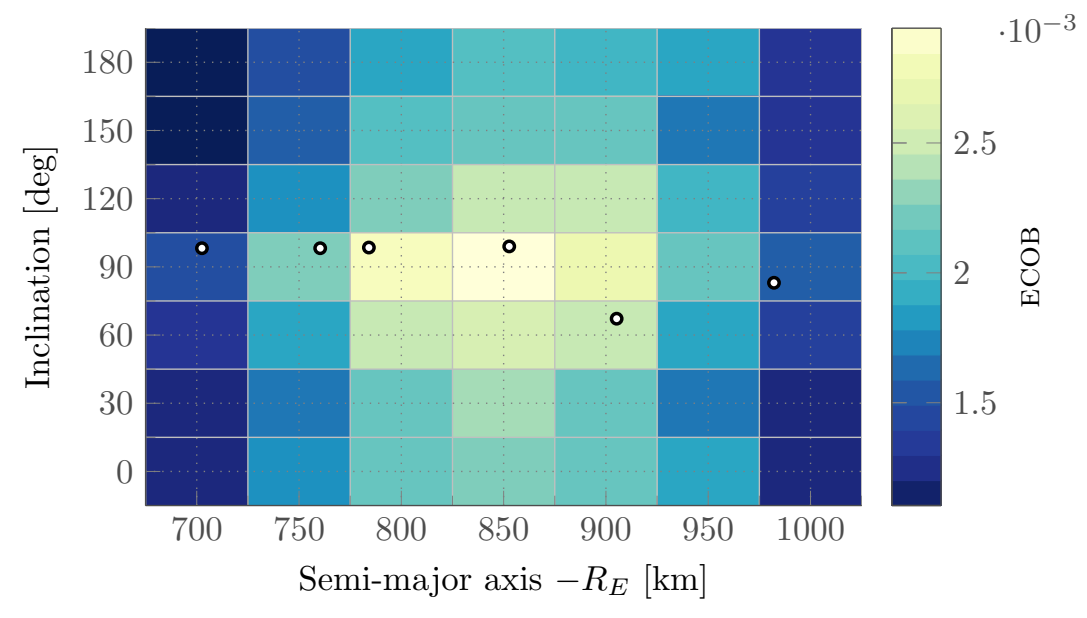

(a) Real targets

25 years

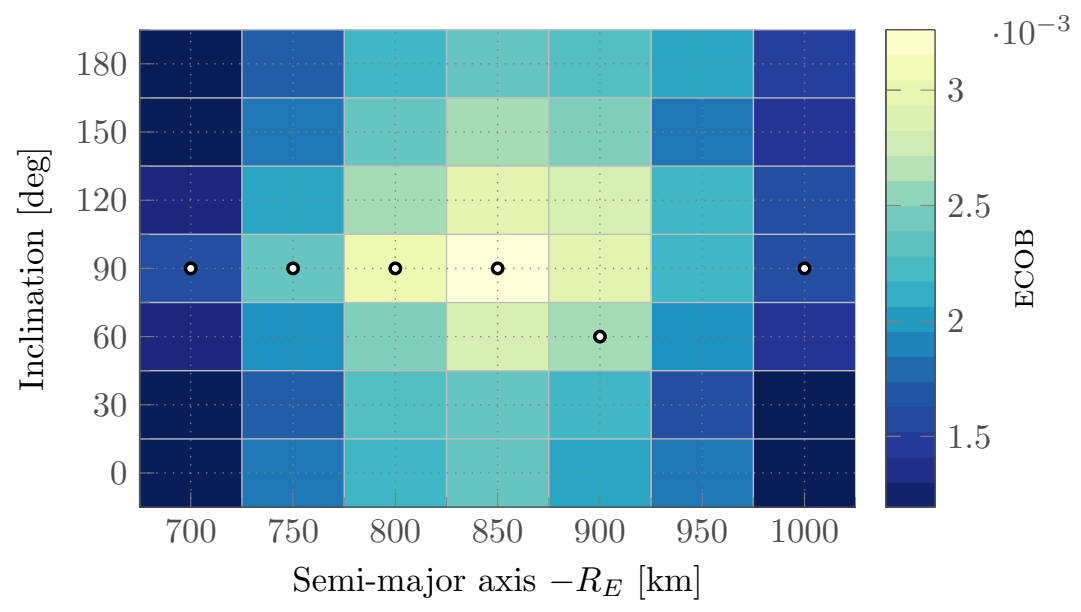

(b) Synthetic targets

Figure 9: Heat map for the criticality index for two different sets of targets. Fragmentation mass equal to $1000 \mathrm{~kg}$. The markers indicate the reference targets. 


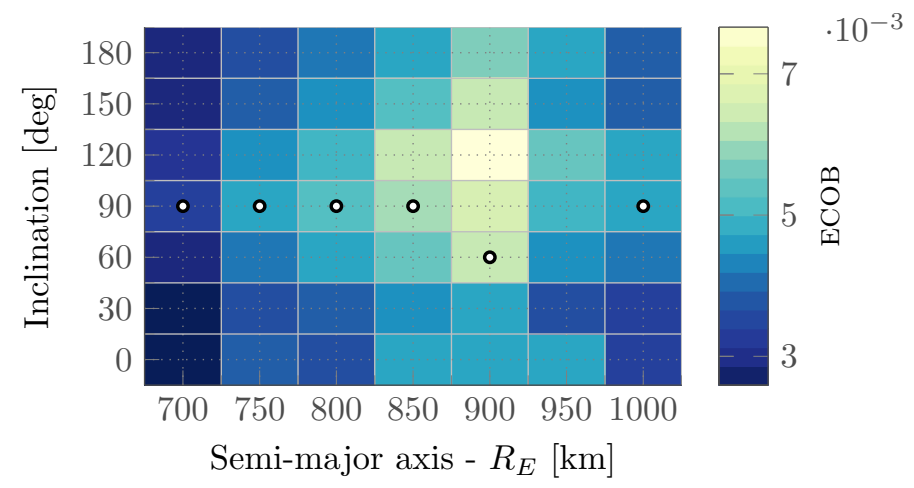

(a) 5 years

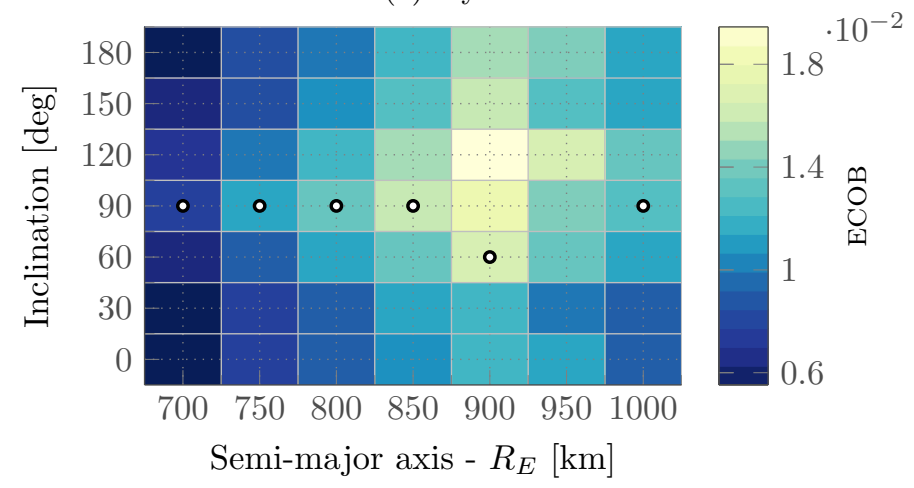

(b) 15 years

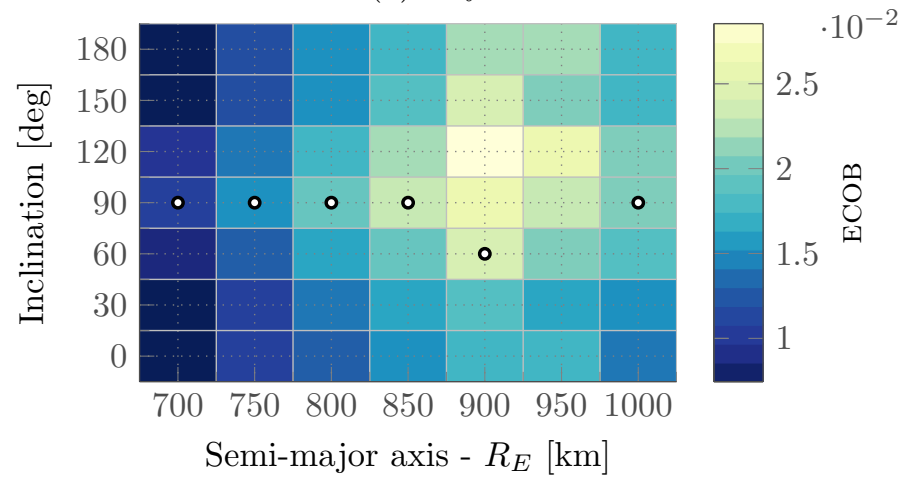

(c) 25 years

Figure 10: Heat map for the criticality index at three different time instants. Fragmentation mass equal to $1000 \mathrm{~kg}$. The markers indicate the reference targets. 
increases. This is expected because if the time of propagation is longer, the fragments at lower altitudes may have the time to decay from the region where the targets are.

\subsection{Effect of the mass}

As explained in Section 3 ECOB depends on the mass of the fragmentation that determines the number of fragments in the debris cloud and its spatial density. One simulation, with propagation time equal to 25 years, was run to evaluate the variation of the index with semi-major axis and breakup mass of the fragmenting object. This setup was chosen to allow the comparison with the Environmental Criticality (EC) by Kebschull et al. (2014b) where the variation of the index with inclination is not considered. For the mass a log-spaced grid was used, with five bins ranging from 100 to $10000 \mathrm{~kg}$. The results of the simulation are shown in Figure 11.

Similarly to Figures $9 \mathrm{~b}$ and $10 \mathrm{c}$, also in Figure 11 the dependence on the semi-major axis is dominated by the distribution of the targets. The dependence on the mass is also easy to explain as when a spacecraft with larger mass fragments, a larger number of objects is produced and a larger collision probability is registered. For comparison, the results obtained with EC by Kebschull et al. (2014b) are shown in Figure 12 It is important to highlight that EC is based on very different principles from the ones used in ECOB, so the comparison can be only qualitative. In fact, the EC measures the change in the collision probability for the whole population (spacecraft, rocket bodies and debris) due to the fragmentation of a specific object, whereas ECOB considers only the collision probability for a selected set of objects representing the active satellites. In addition, in EC, several different fragmentation epochs are considered for a breakup, whereas in ECOB the effect of time is not present because the background debris population is not considered. Finally, EC takes into account the feedback effect, which is when a fragmentation triggers other new breakups, as

\footnotetext{
${ }^{2}$ Jan Siminski, Graduate Trainee, ESA, personal communication, 19/06/2015
} 


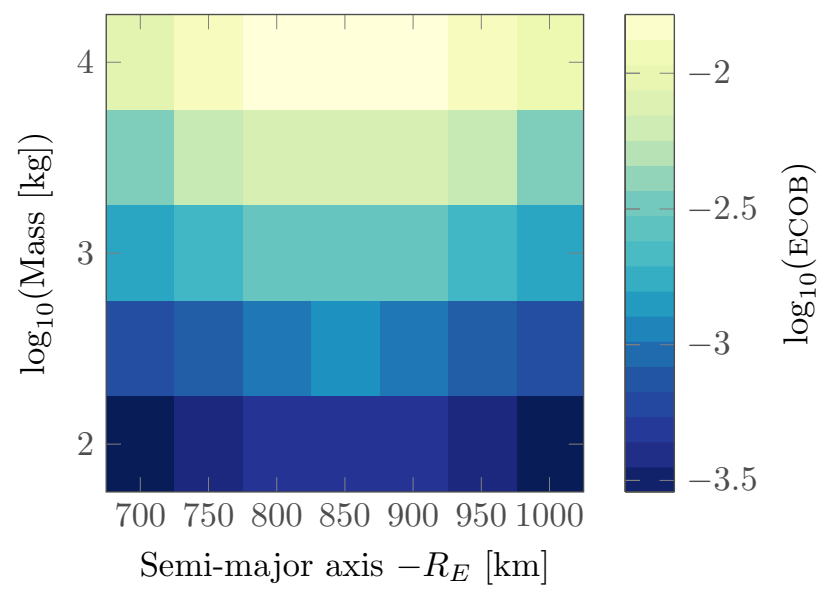

Figure 11: Heat map for ECOB as a function of the semi-major axis and the fragmenting mass.

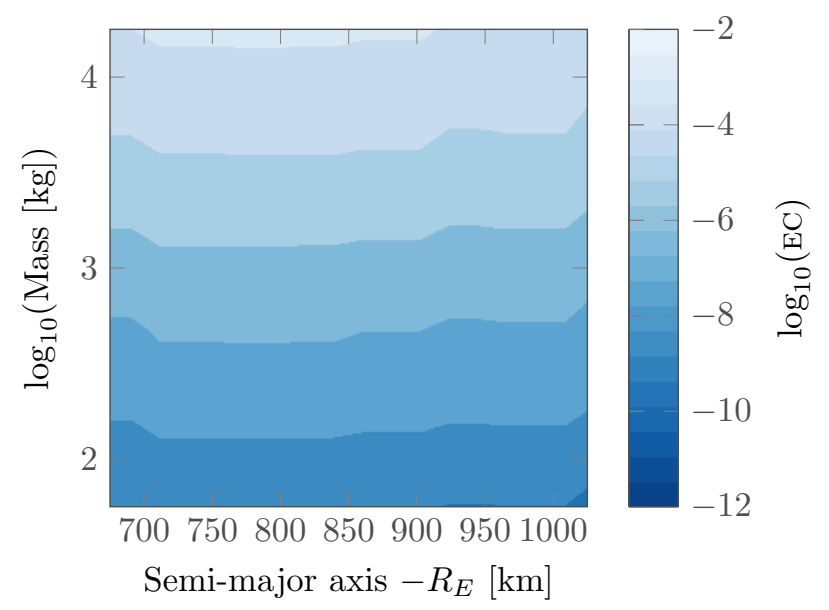

Figure 12: Heat map for EC as a function of the semi-major axis and the fragmenting mass. Figure by Jan Siminski. 
the debris environment evolution is studied with the model SANE (Kebschull et al. 2014a), whereas ECOB describes the interaction of a single fragment cloud with the selected targets. Besides these differences, Figure 12 shows a similar dependence on the mass and on the semi-major axis. If the feedback effect were dominant in this time scale, then Figure 12 would show a stronger dependence on the mass and a dependence on the semi-major axis driven by the altitude and not by the target distribution. This suggests that the feedback effect is not particularly relevant for simulation of 25 years.

A closer look on the results in Figure 11 reveals that the dependence of the index ECOB on the mass $M$ is predictable. Figure 13 shows the dependence of the index on the mass for different altitudes and one can observe how the curve is a straight line in the log-log representation. In particular, it can be found that

$$
\operatorname{ECOB}(M)=\left(\frac{M}{M_{\text {ref }}}\right)^{0.75} \cdot \operatorname{ECOB}\left(M_{\text {ref }}\right)
$$

where the exponent 0.75 is a direct results of the NASA breakup model. In fact, according to the model (Johnson and Krisko, 2001), the number of produced fragments larger than a given characteristic length is equal to

$$
N_{f}\left(L_{c}\right)=0.1(M)^{0.75} L_{c}^{-1.71}
$$

with $M$ equal to the sum of the target mass and the projectile mass in the case of catastrophic collisions.

\section{Index computation}

The observation in Equation 19 is important because it allows the required number of simulations to be reduced, simplifying the computation of the index for a generic object. In fact, the index can be computed through simulations using a single reference value for the mass (i.e. $10000 \mathrm{~kg}$ ) and a grid in semi-major axis and inclination. 


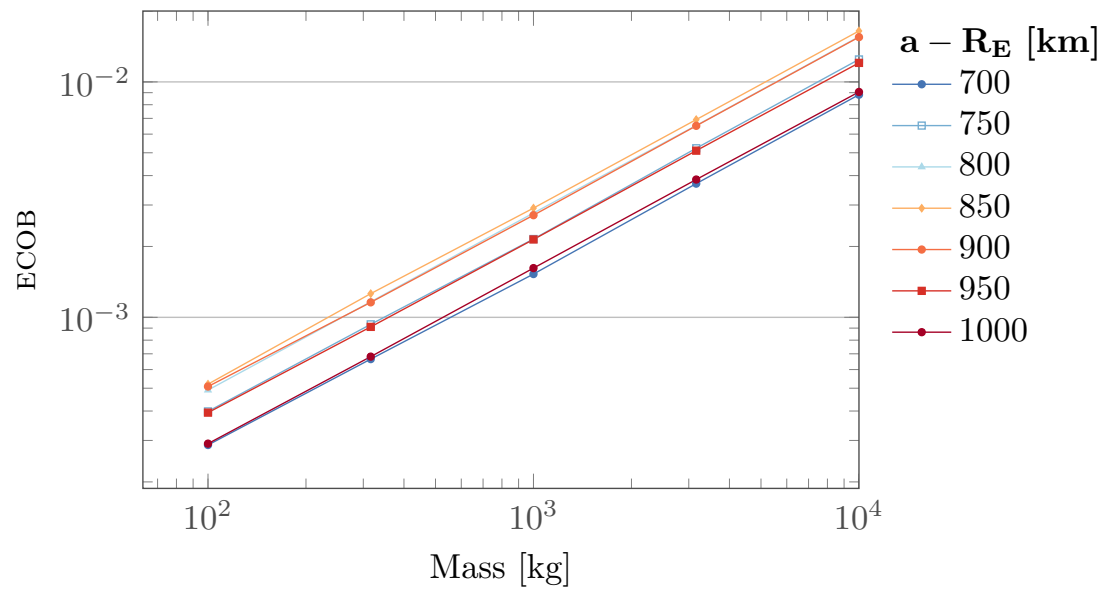

Figure 13: Index dependence on the mass for different altitudes.

\subsection{Post-processing}

The value of the index on the plane of semi-major axis and inclination, with a fixed value of mass, can be stored and scaled to the value of the mass of the analysed fragmenting object. Then, finding the value of the index at the semi-major axis and inclination of the analysed object becomes a problem of fitting the surface defined by the value of ECOB in the grid of semi-major axis and inclination. The advantage of this approach is that the computational effort is required only to generate the surface (which as described in Section 3 is assumed to be slowly-changing if the development of space activities does not change abruptly). In this way, the reference surface is computed and stored, and severity index for all the objects in a database, such as DISCOS, can be quickly computed in post-processing, with a fitting procedure. Given the pairs $\left\{\left(\mathbf{x}_{i}^{*}, z_{i}^{*}\right)\right\}_{i}$, where $\mathbf{x}_{i}^{*}$ is a point of the grid in semi-major axis and inclination and $z_{i}^{*}$ is the corresponding value of $\mathrm{ECOB}$, the goal is to use these values to define a surface $Z=\left\{\left(\mathbf{x}_{i}, z_{i}\right)\right\}_{i}$ defined on the whole domain. Three options for the fitting were identified and tested with MATLAB.

- interpolation (Figure 14), which finds the value $z_{i}$ at each point $\mathbf{x}_{i}$ of the domain using the values $z_{i}^{*}$ at the nearest grid points; the number of the considered near grid points depends on the interpolation scheme (e.g. 
Table 1: Methods for surface fit.

\begin{tabular}{rrrr}
\hline Method & Adj. $R^{2}$ & Storable & Parametric \\
\hline Interpolation & $(1)$ & $($ Yes $)$ & $($ Yes $)$ \\
Local regression smoothing & 0.9966 & (Yes) & No \\
Polynomial & 0.9687 & Yes & Yes \\
\hline
\end{tabular}

bilinear, bicubic, biharmonic) and in any case the value of the surface at the grid points is equal to the initial values, so that $Z\left(x_{i}^{*}\right)=z_{i}^{*}$;

- local regression smoothing methods (Figure 15), which uses least squares regression techniques in combination with a weighting function that gives larger importance to closer data points when computing the value $z_{i}$ of a generic point $\mathbf{x}_{i}$ in the domain ${ }^{3}$ in this case is not assured that $Z\left(x_{i}^{*}\right)=z_{i}^{*}$;

- polynomial curve (Figure 16), which fits the data with a polynomial function in two dimensions, up to degree five.

In the three figures, the black dots represent the computed values of the index whereas the coloured surface is obtained by the different fitting methods. In all cases, representative targets are used, for a propagation time of 25 years and a fixed value of the fragmenting mass equal to $1000 \mathrm{~kg}$.

The main features of the comparison are summarised in Table1. In the case of interpolation, MATLAB offers different options for the curve to use (e.g. linear, cubic, or bi-harmonic spline): in any case the adjusted $R^{2}$ is obviously equal to one as $Z\left(x_{i}^{*}\right)=z_{i}^{*}$, so this cannot be used as a measure of the quality of the fit. Figure 14 shows in any case how, even with a coarse grid, the interpolation provides a good representation of the surface. The interpolation does not give a strictly parametric expression of the surface $Z$, but analytical methods can be easily implemented to describe the surface. When matLaB is used, the output of the fit can be stored in a special type of variable and reused to compute the index for different objects. When MATLAB is not used, it may be necessary

${ }^{3}$ A description of the method can be found at http://uk.mathworks.com/help/curvefit/ smoothing-data.html last access 5 January 2016. 


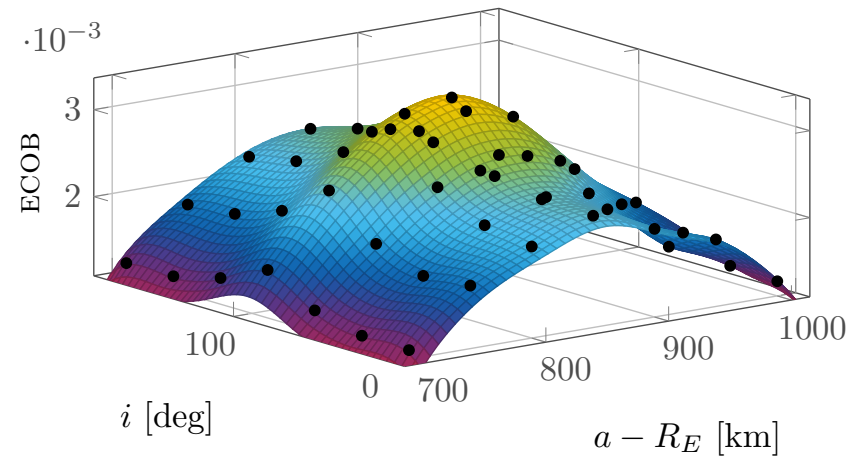

Figure 14: Index interpolation with biharmonic spline.

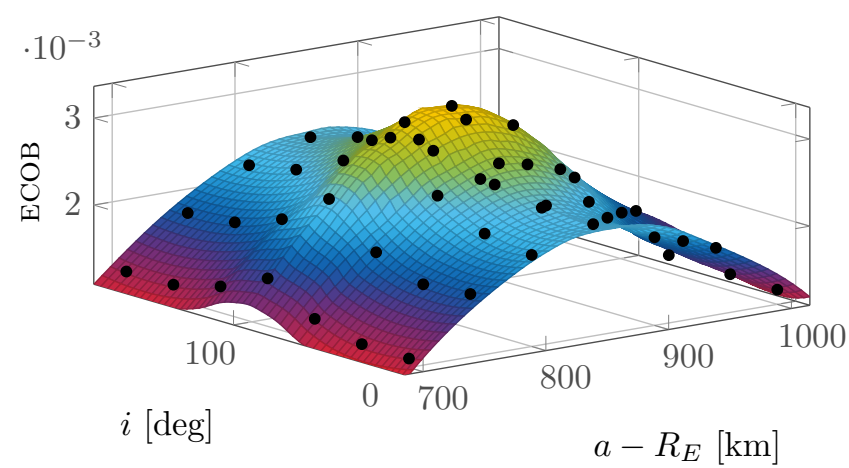

Figure 15: Index fit through a local regression smoothing method.

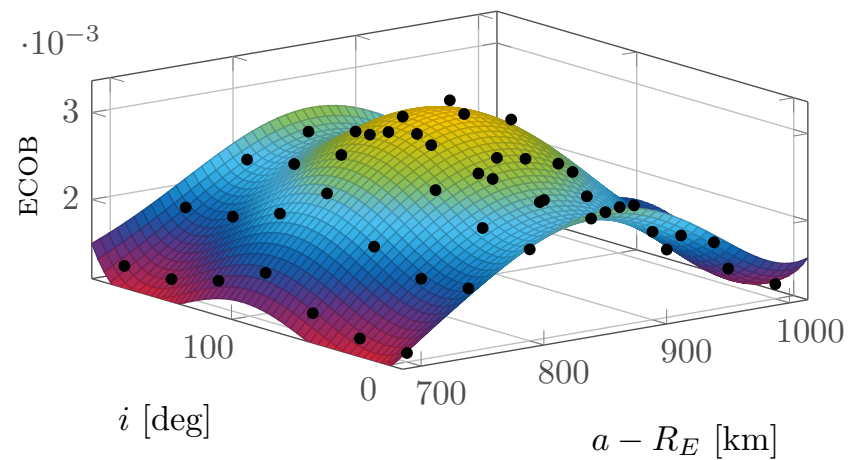

Figure 16: Index fit to a polynomial curve. 
to recompute the local interpolation for each object, but the operation is not expected to be computationally expensive.

When regression methods are applied, the whole shape of the surface is described. In this case MATLAB offers two options for the regression model: a linear or a quadratic one. The value of the adjusted $R^{2}$ is good, but this method is not parametric, has a more complex formulation and does not seem to offer any additional advantage compared to the interpolation. For this reason, the regression methods were discarded.

The third method, the polynomial curve, combines the positive features of the two previous approaches. Firstly, it describes the whole curve, so the coefficients needs to be computed only once and then the equation can be applied for the computation of the index for any object. Secondly, once the equation is obtained, it is completely independent from any programming language. However, the performance in terms of adjusted $R^{2}$ were not considered sufficient. Figure 16 shows the result for a polynomial curve of fifth order both in $x$ and $y$ : the polynomial representation appears to smooth the curve, especially at the peak. For these reasons, the local interpolation was preferred.

\subsection{Tool structure}

The observations in the previous sections help defining the structure of the tool to compute the environmental index. This can be divided into two parts: first, the computation of index using a set of targets and a reference mass for the fragmentation on a predefined grid in semi-major axis and inclination; second, the computation of the index for different fragmenting spacecraft and rocket bodies.

The first part of the tool uses the method based on the continuity equation and it is highly computationally expensive, so that the super-computer facilities of the University of Southampton, IRIDIS, are required. Once this phase is concluded, the output consists in the matrix plotted in Figure 9, which can be easily saved and exported in different formats (e.g. ASCII file), depending on the user platform. The matrix represents the input of the code that actually 
computes the environmental index for the objects of interest. The code performs the fitting of the surface using a local interpolation method and rescaling the index depending on the mass of the studied objects. The code can receive as an input a file containing the list of the objects to analyse. For example, the results in Section 6 are obtained using as a database the data extracted from DISCOS (Flohrer et al. 2013) considering objects in orbits between 700 and $1000 \mathrm{~km}$. For each object its kind (i.e. rocket body, spacecraft, other) and its year of launch are specified, so that the user can choose to study only a subset of the list. A maximum number of the objects to study can also be specified.

\subsection{Computational time}

The tests with the coarse grid in Section 4 were used also to assess the computational time required by the simulations and identify the most effective parallelisation strategy to run cases with fine grids. Figure 17 shows the computational time for the cases as in Figures 9. The computational time refers to a machine with $4 \mathrm{CPU}$; the whole code is written in MATLAB and exploits its built-in parallel statements (e.g. parfor). The histogram in Figure 17 shows the computational time for the three main functions in the code

- propTarget propagates the target trajectories for the desired time frame considering drag and $J_{2}$;

- buildLayer simulates a catastrophic collision for a given breakup mass in each cell and compute the cloud spatial density, including fragments down to $1 \mathrm{~cm}$, for the whole desired time window

- addCollProb computes the collision probability for each target and the value of the index on the studied grid applying a weighting factor, if specified.

Applying the same settings to compute a case with a fine grid as the one used in Section 6 would require more than 22 hours of computation with $4 \mathrm{CPU}$ (that is 3.6 days in CPU time) assuming that the simulation is still manageable in terms of RAM. 


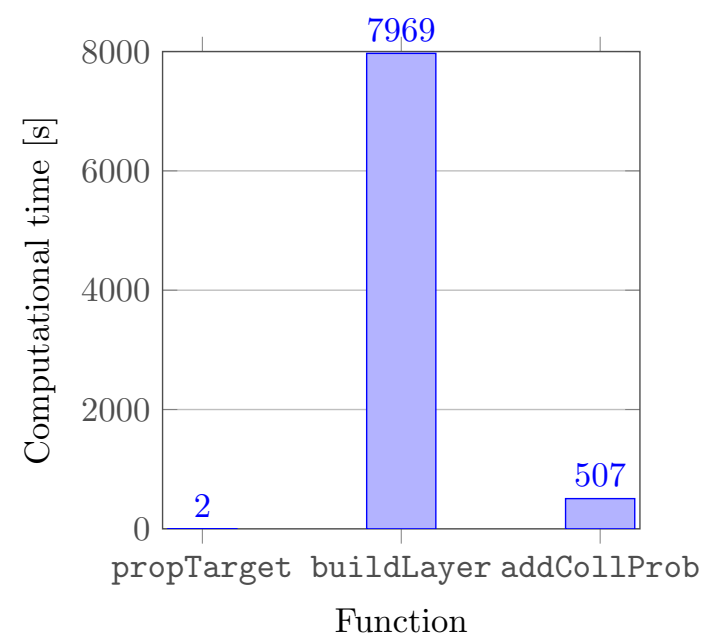

Figure 17: Computational time on a PC with $4 \mathrm{CPU}$ for a layer with coarse grid.

For this reason, it was decided to use IRIDIS, the super-computer facilities at the University of Southampton, to run the simulations with fine grids. The computation of one layer was divided into columns (simulations with the same semi-major axis), which are launched as separate jobs, each one with 12 processors allocated (the maximum). In this way one exploits not only the parallel features in MATLAB, but also the possibility of running multiple jobs at the same time. The submission of the jobs is fully automatised with a simple bash script. The whole setting allows obtaining a full layer in a period of time between one and three hours (depending on the availability of processors on the server). The real computational time (summing the running time of each job) would be around 19 hours (equal to almost six days of CPU time).

\section{Results}

Once the structure of the index was set, some simulations with a fine grid in semi-major axis and inclination were run. In this case, the grid is spaced of $10 \mathrm{~km}$ in semi-major axis and 10 degrees in inclination. The same grid is used both for the generation of the fragmentations and for the selection of the representative targets. Using, as before, the threshold of $90 \%$ for the represented 
$A_{c}, 15$ targets are identified, as shown in Figure 18. These targets are derived from the data from DISCOS considering only spacecraft (no rocket bodies) in orbit between 700 and $1000 \mathrm{~km}$, and launched in the last ten years. The parameters of these targets are listed in Table 2 A reference breakup mass equal to $10000 \mathrm{~kg}$ was used. A propagation time of 25 years was considered.

As already presented in Figure 6, the cross-sectional area is not uniformly distributed across the whole LEO region: active satellites are mostly concentrated in polar orbits and this explains the distribution of the targets in Figure 18 The concentration of targets at latitudes around 80 degrees results in a much higher index in these regions. For fragmentations at these latitude, the targets can spend a large part of their orbits in the area (the extremes of the band) where the spatial density is maximum. The map appears quite symmetrical with respect to $i=90$ degrees, so the possibility of reducing the grid in inclination to $\left[0,90^{\circ}\right]$ may be investigated in the future. For what concerns the semi-major axis, a high density of targets around 800-850 km makes this region the one with the highest environmental index.

The map in Figure 18 was combined with a database generated from DISCOS that contains all objects in orbits between 700 and $1000 \mathrm{~km}$ to evaluate the environmental index of objects already in orbit. Note that in this case, differently from the list used to define the target set, there is no filter on the launch date, and both rocket bodies (RB) and payloads (PL) are present. In this way, the index can be used to assess the environmental impact of both failed/not planned disposal strategies for payloads and of not sufficient passivation measures for rocket bodie:4

As in Figure 18, Figure 19 represents the value of ECOB computed for a reference mass equal to $10000 \mathrm{~kg}$ and propagation time equal to 25 years, but in this case a contour plot is used to represent the surface obtained with the

\footnotetext{
${ }^{4}$ The failure of a rocket body due to insufficient passivation measures may be modelled more accurately by an explosion than by a collision. The two classes of fragmentation produce fragments with a different distribution in size and in energy. For this reason, future work may investigate the difference in ECOB if explosions are modelled instead of catastrophic collisions.
} 


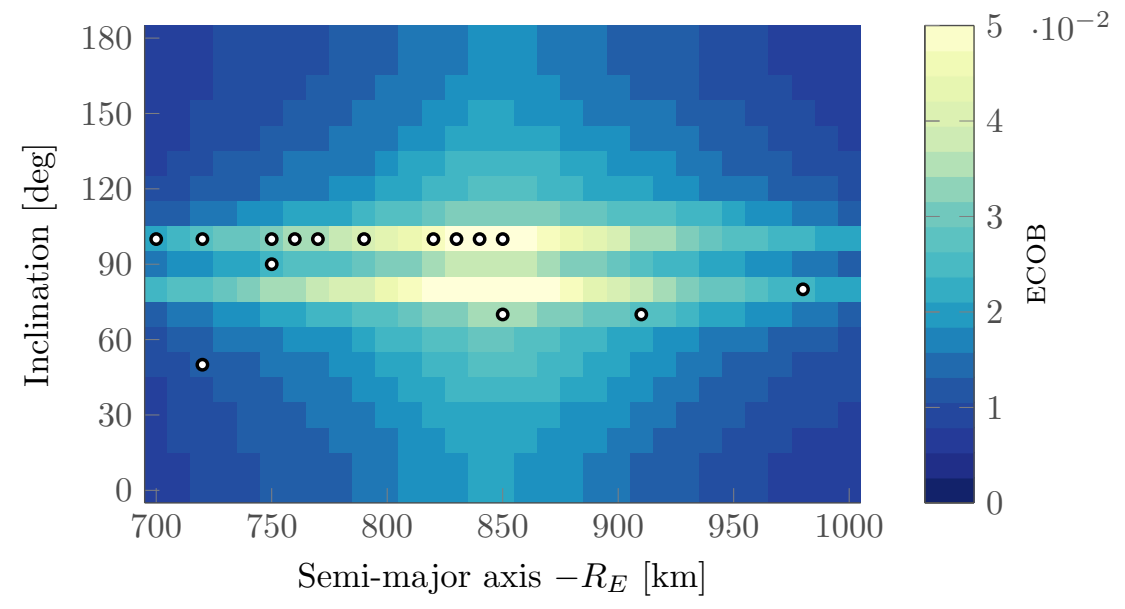

Figure 18: Resulting index for catastrophic collisions with breakup mass equal to $10000 \mathrm{~kg}$. Collision probability measured on the 15 targets indicated by a marker. Propagation time equal to 25 years.

Table 2: Parameters of the 15 representative targets. $\bar{h}=a-R_{E} ; \bar{M}$ and $\bar{A}_{c}$ refer to average value in the cell.

\begin{tabular}{rrrrrr}
\hline ID & $\bar{h}[\mathrm{~km}]$ & $i[\mathrm{deg}]$ & $\bar{M}[\mathrm{~kg}]$ & $\bar{A}_{c}\left[\mathrm{~m}^{2}\right]$ & $w$ \\
\hline 1 & 820 & 100 & 1533 & 14.45 & 0.2310 \\
2 & 700 & 100 & 1332 & 15.48 & 0.1237 \\
3 & 720 & 100 & 3048 & 9.81 & 0.0784 \\
4 & 850 & 100 & 1298 & 9.41 & 0.0752 \\
5 & 770 & 100 & 1705 & 11.22 & 0.0717 \\
6 & 830 & 100 & 1820 & 11.05 & 0.0706 \\
7 & 910 & 70 & 7000 & 16.22 & 0.0519 \\
8 & 760 & 100 & 1054 & 12.63 & 0.0404 \\
9 & 790 & 100 & 436 & 2.35 & 0.0301 \\
10 & 750 & 100 & 523 & 5.24 & 0.0251 \\
11 & 750 & 90 & 605 & 15.71 & 0.0251 \\
12 & 850 & 70 & 3250 & 15.21 & 0.0243 \\
13 & 840 & 100 & 2200 & 15.10 & 0.0241 \\
14 & 720 & 50 & 172 & 2.87 & 0.0183 \\
15 & 980 & 80 & 820 & 10.78 & 0.0172 \\
\hline & & & & &
\end{tabular}




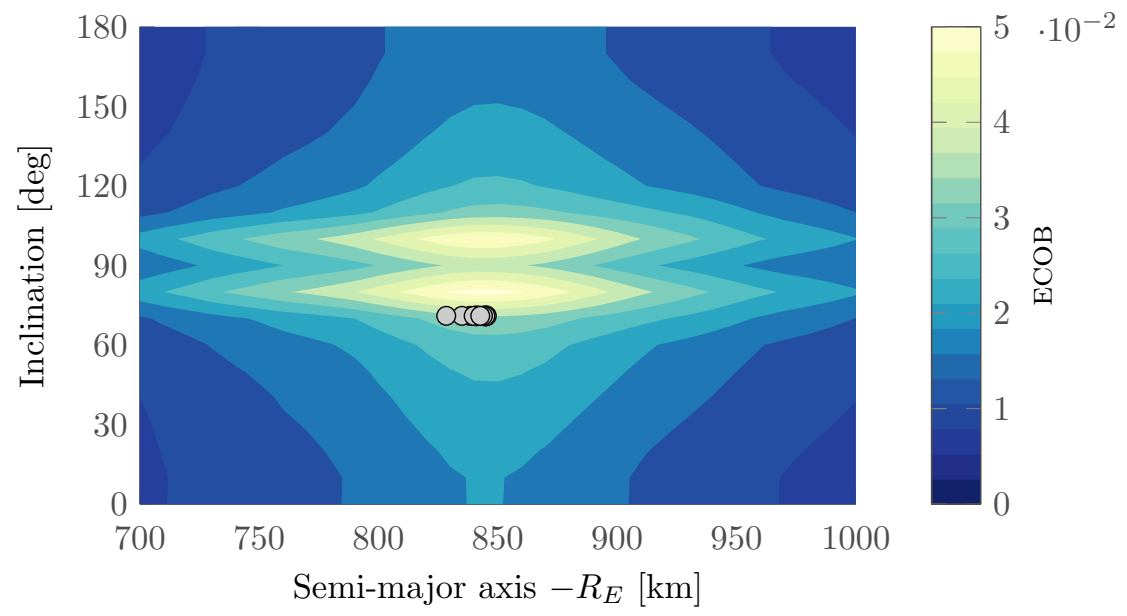

Figure 19: Contour plot of the environmental index for a breakup mass equal to $10000 \mathrm{~kg}$ and propagation time of 25 years. The markers indicate the ten objects, from DISCOS data, with the highest environmental index. The size of the marker is proportional to the mass of the object.

Table 3: Top ten objects with the largest environmental index ECOB among DISCOS data considering all objects in orbits between 700 and $1000 \mathrm{~km} . \bar{h}=a-R_{E}$.

\begin{tabular}{cccccccc}
\hline ID & COSPAR & Name & Type & $\bar{h}[\mathrm{~km}]$ & $i[\mathrm{deg}]$ & Mass $[\mathrm{kg}]$ & ECOB \\
\hline 2 & $2004-021 \mathrm{~B}$ & SL-16 R/B & RB & 845 & 70.9997 & 9000 & 0.032896 \\
3 & $2007-029 B$ & SL-16 R/B & RB & 845 & 70.9753 & 9000 & 0.032837 \\
1 & $2000-006 B$ & SL-16 R/B & RB & 841 & 71.0029 & 9000 & 0.032679 \\
7 & $1990-046 B$ & SL-16 R/B & RB & 844 & 70.9989 & 8226 & 0.030711 \\
5 & $1992-093 B$ & SL-16 R/B & RB & 842 & 71.0226 & 8226 & 0.030682 \\
10 & $1993-016 B$ & SL-16 R/B & RB & 843 & 71.0068 & 8226 & 0.030667 \\
9 & $1988-102 B$ & SL-16 R/B & RB & 840 & 71.0004 & 8226 & 0.030476 \\
4 & $1985-097 B$ & SL-16 R/B & RB & 838 & 71.0039 & 8226 & 0.030420 \\
6 & $1987-041 B$ & SL-16 R/B & RB & 835 & 71.0084 & 8226 & 0.030223 \\
8 & $1988-039 B$ & SL-16 R/B & RB & 828 & 71.0160 & 8226 & 0.029394 \\
\hline
\end{tabular}


interpolation. The markers indicate the ten objects with the highest environmental index among the ones in the database. In particular, the location of the marker indicates the object orbital parameters and the size of the markers is proportional to the object mass. In this case, the markers have all similar size because all objects belong to the same family as shown in Table 3 . The objects in Table 3 are sorted by the value of the environmental index ECOB, whereas the ID in the first column is related to the object mass, with ID $=1$ for the most massive object in the database. All objects in Table 3 belong to the same family, SL-16 R/B: they combine high mass and orbits within the most critical regions.

The same analysis was performed also considering only spacecraft launched at least ten years ago. The results are shown in Table 4 and Figure 20. Also in this case, most objects belong to the same family (Cosmos satellites) with the exception of Envisat. It presents a much larger environmental index due to its large mass.

From these two examples, the proposed environmental index appears able to give insight into the effect of different breakups on active satellites. A further formalisation of the index may include levels such as the severity number defined in (European Cooperation for Space Standardisation, 2009) to evaluate different failure modes of missions. In that case, four levels of severity are defined (i.e. catastrophic, critical, major, negligible) that could be related to the value of the environmental index. Observe that the connection between the environmental index and the severity levels cannot be done by setting a simple numerical threshold for the index. In fact, the absolute value of the index will change every time that the target set is redefined. The connection to the severity levels may be done by identifying for each level a reference fragmentation (e.g. the breakup of Envisat to represent a catastrophic level of severity). When another space object is evaluated, its index is compared to the ones of the reference fragmentations to assess its level of severity. This would also give an immediate meaning to the value of the index. The definition of such a scale will be investigated in future work. 


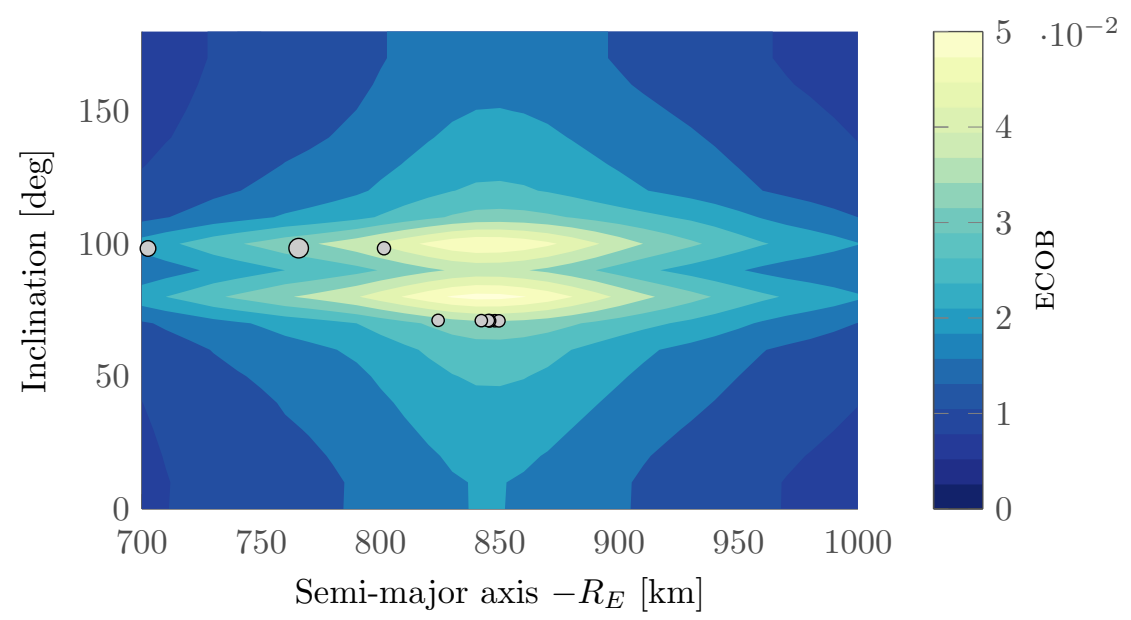

Figure 20: Contour plot of the environmental index for a breakup mass equal to $10000 \mathrm{~kg}$ and propagation time of 25 years. The markers indicate the ten objects with the highest environmental index, considering only spacecraft launched more than ten years ago. The size of the marker is proportional to the mass of the object.

Table 4: Top ten objects with the largest environmental index ECOB among DISCOS data considering only spacecraft launched more than ten years ago and in orbits between 700 and $1000 \mathrm{~km} . \bar{h}=a-R_{E}$.

\begin{tabular}{cccccccc}
\hline ID & COSPAR & Name & Type & $\bar{h}[\mathrm{~km}]$ & $i[\mathrm{deg}]$ & Mass $[\mathrm{kg}]$ & ECOB \\
\hline 20 & 2002-009A & Envisat & PL & 766 & 98.3338 & 8111 & 0.028247 \\
32 & 2002-056A & Cosmos 1656 & PL & 801 & 98.2865 & 3680 & 0.018946 \\
39 & $1990-046 \mathrm{~A}$ & Cosmos 2082 & PL & 845 & 71.0418 & 3221 & 0.015260 \\
36 & $1984-106 \mathrm{~A}$ & Cosmos 1603 & PL & 846 & 71.0240 & 3221 & 0.015245 \\
34 & $1985-097 \mathrm{~A}$ & Cosmos 1697 & PL & 848 & 70.9634 & 3221 & 0.015166 \\
40 & $1988-039 \mathrm{~A}$ & Cosmos 1833 & PL & 842 & 71.0020 & 3221 & 0.015163 \\
38 & $1987-041 \mathrm{~A}$ & Cosmos 1844 & PL & 846 & 70.9001 & 3221 & 0.015133 \\
37 & $1987-027 \mathrm{~A}$ & Cosmos 1943 & PL & 850 & 70.9178 & 3221 & 0.015092 \\
35 & $1985-042 \mathrm{~A}$ & Terra & PL & 824 & 71.1123 & 3221 & 0.014349 \\
25 & $1999-068 \mathrm{~A}$ & Adeos 2 & PL & 703 & 98.2107 & 5190 & 0.013461 \\
\hline
\end{tabular}


It is also important to highlight the limitations of the proposed index. The propagation method used for the fragment clouds considers only the effect of drag, whereas at altitudes above $800 \mathrm{~km}$ solar radiation pressure becomes relevant too. An improvement in the propagation method could provide more accurate results on the cloud propagation. In addiction, the propagation method limits the extent of the analysis both in altitudes $(700-1000 \mathrm{~km})$ and in time (maximum 25 years). Finally, no feedback effect can be considered with the current formulation. Some of these aspects are considered in alternative environmental indices, to which ECOB is compared in the next section.

\section{Comparison with other formulations}

The proposed index ECOB was compared to the other environmental index formulations developed in the framework of the ESA study Fragmentation Consequence Analysis for LEO and GEO Orbits.

\subsection{Comparison with $\mathrm{EC}$ and $\mathrm{FOM}$}

As already mentioned, EC is the index developed by Kebschull et al. (2014b). EC is based on the computation of the change in the collision probability for the whole debris population due to the fragmentation of a selected object. A catastrophic collision is simulated at different time instants within the considered time window and its effect on the global debris population is obtained by applying an analytical model of the debris evolution, SANE. The proposed index of criticality takes into account both the consequences of the fragmentation $c_{\text {impact }}$ and its probability of happening $c_{\text {risk }}$

$$
\mathrm{EC}=c_{\text {risk }} \cdot c_{\text {impact }}=\sum_{t=t_{0}}^{t_{f}}\left[\left(c_{\text {risk }}\right)_{t} \cdot\left(c_{\text {impact }}\right)_{t}\right],
$$

where $t_{0}$ and $t_{f}$ are the extremes of the considered time interval. The probability of a fragmentation happening is computed as

$$
\left(c_{\text {risk }}\right)_{t}=\Phi A_{c}\left(t-t_{0}\right),
$$


with $\Phi$ flux of the background debris population on the object's orbit. The value of $\Phi$ is obtained with SANE as the product of the debris density at the object's orbit and the relative velocity between the objects and the debris population. The effect of the fragmentation is computed as

$$
\left(c_{\text {impact }}\right)_{t}=\sum_{t=t_{\text {frag }}}^{t_{\text {end }}}(\Delta p) \Delta \tau=\sum_{t=t_{\text {frag }}}^{t_{\text {end }}}\left(\Phi_{\text {frag }}-\Phi_{\text {no frag }}\right) A_{c}\left(t-t_{\text {frag }}\right),
$$

where $\Phi_{\text {frag }}$ and $\Phi_{\text {no frag }}$ are respectively the flux in the scenario with the studied fragmentation and the flux without the fragmentation and $\Delta \tau=t_{\text {end }}-t_{\text {frag }}$.

Radtke et al. (2014) compares EC with an alternative index developed by AIRBus (Utzmann et al., 2012). The index by AIRBus, called Figure Of Merit (FOM), is computed as

$$
\mathrm{FOM}=\Phi A_{c} M^{0.75} \Delta t_{\mathrm{orb}}
$$

where $\Phi$, in this case with units $\left[\mathrm{m}^{-2} \mathrm{yr}^{-1}\right]$, is the debris flux in the object's orbit, $A_{c}$ and $M$ are respectively the cross-sectional area and the mass of the object, $\Delta t_{\text {orb }}[\mathrm{yr}]$ is the remaining orbital lifetime.

The proposed index ECOB was computed for the top 20 objects with the highest criticality according to EC (Radtke et al., 2014): the list of objects is reported in Table 5 and for each object the criticality estimated by the three methods is shown. Note that it is more interesting to study the ranking obtained with the different approaches more than the numerical value of the indices for the studied cases. For this reason, the results were analysed studying the correlation among the methods. Firstly, Figure 21 shows the correlation between the proposed index ECOB and FOM. The results show a good coherence between the two methods except for the case of Envisat and MetOp-A. For Envisat, the large difference may be explained by the fact that FOM takes into account the exposure of the object to the background population $\left(\Phi A_{c}\right)$. This term is particularly relevant for Envisat as its orbit is within the most affected region by the Iridium-Cosmos fragmentation. On the other hand, the value for MetOp-A 


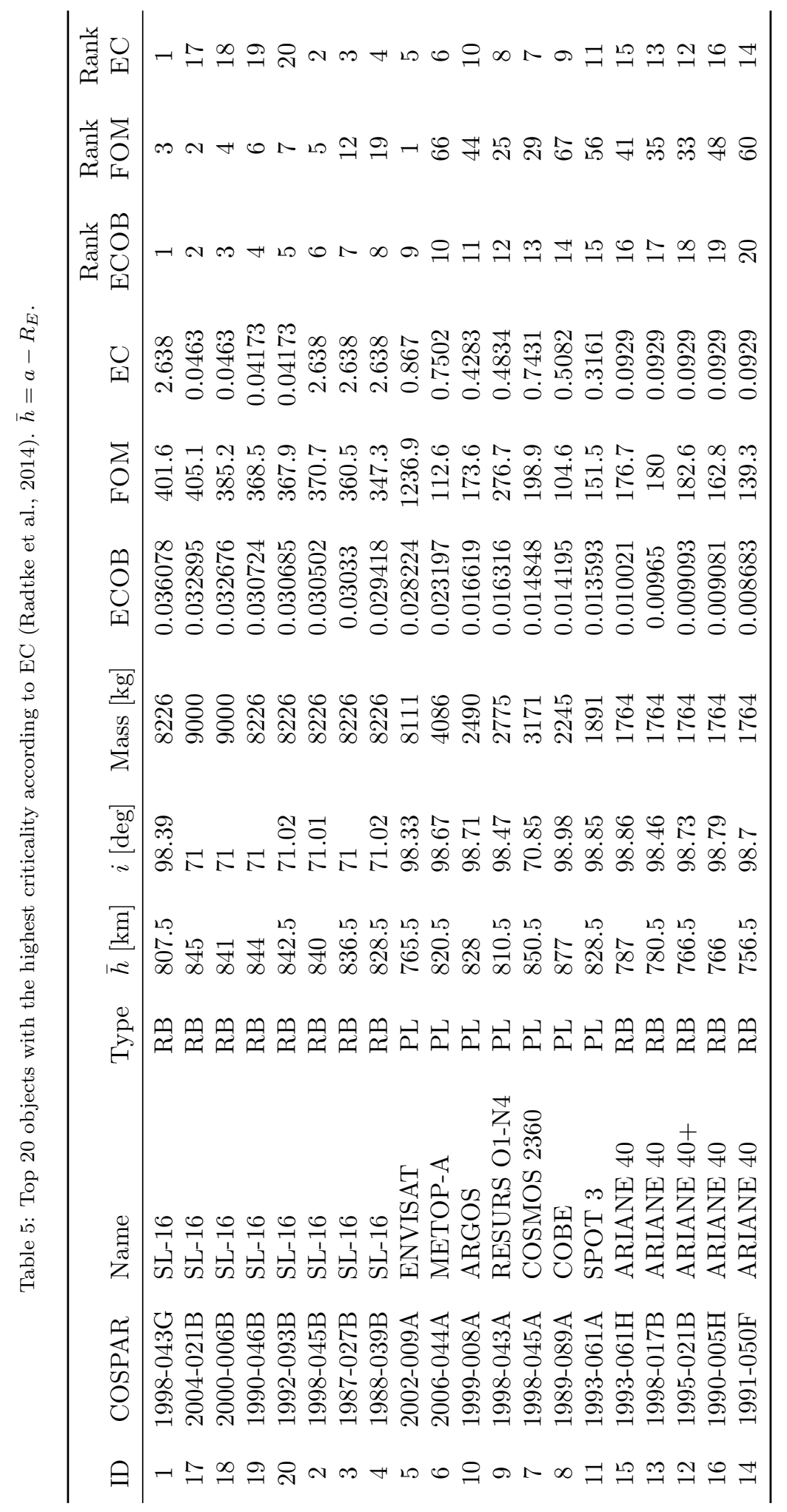




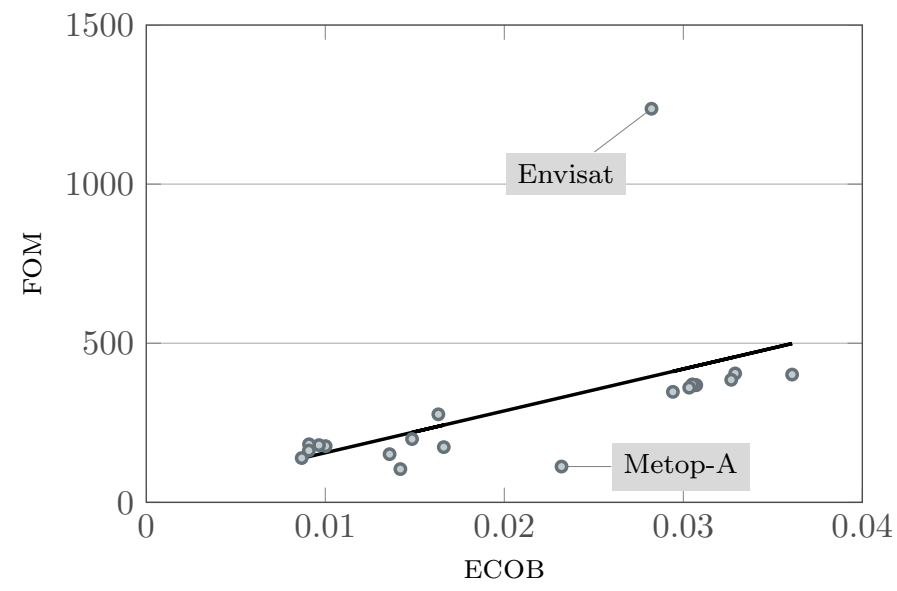

Figure 21: Correlation between the proposed index ECOB and FOM.

may be explained by the fact that, as it is an operational spacecraft, in FOM its orbital lifetime is computed as

$$
\Delta t_{\text {orb }}=\Delta t_{\text {act }}+25
$$

where $\Delta t_{\text {act }}$ is the remaining mission duration. In this way, its orbital lifetime results much shorter than Envisat, even if MetOp-A is on a orbit with higher altitude. The distinction between active and not-active satellites is not trivial; moreover, one can be interested in evaluating the environmental impact of a spacecraft without considering post-disposal manoeuvres exactly to assess how necessary those manoeuvres are. For this reason, active and inactive objects are treated in the same way in the computation of ECOB.

Figure 22 shows the analysis on the correlation between EC and the proposed index ECOB. Also in this case it is important to remark that the two indices rely on very different models. In both cases it is true that not only the mass plays an important role in the value of the index, but also the orbital region. However, for ECOB this refers to the interaction with the reference targets, whereas for EC also the effect of the background population is considered. Observing the results in Figure 22 there is an evident difference in the evaluation of the 


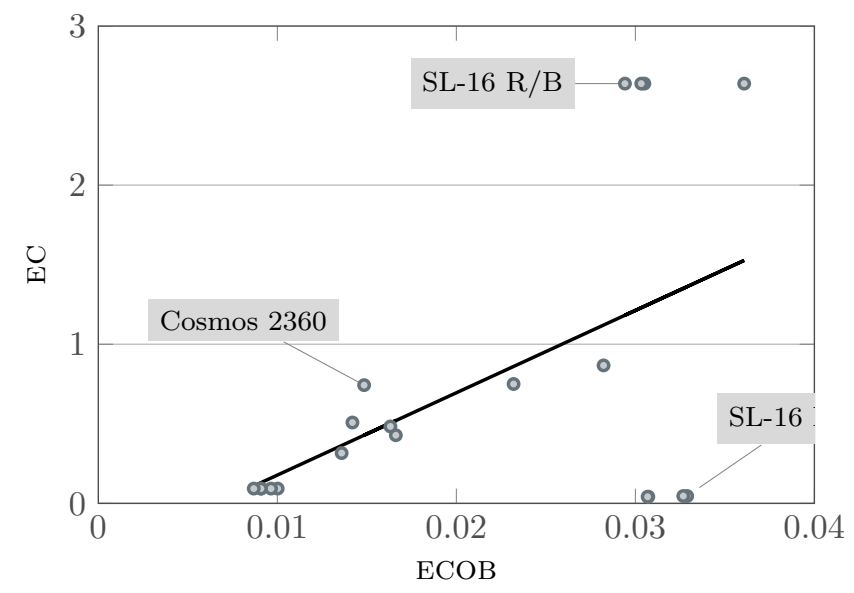

Figure 22: Correlation between the proposed index ECOB and *EC.

objects with ECOB between 0.03 and 0.04. These objects are all SL-16 R/B with similar mass and similar orbits. For this reason, their ECOB (and also their FOM) is similar. However, the ranking with EC is very different, with four objects with the highest criticality and the other ones with the lowest criticality among all the 20 tested objects. This result is explained by the lack of interpolation between cells in the evaluation of the background population within SANE (Radtke et al., 2014). A small difference is present also in the evaluation of Cosmos 2360: with ECOB this object is less critical than objects with smaller mass (i.e. Argos and Resurs O1-N4) because its inclination is such that it is not in the most critical area. On the other hand, EC does not consider the dependence on the inclination, so the object is ranked 7 th versus 13 th with ECOB.

It can be concluded that ECOB gives consistent results as a small change in the orbit results in a small change in ECOB. This does not happen with $\mathrm{EC}$, but the behaviour can be easily fixed by implementing the interpolation of the background population. The dependence on the inclination, not present in FOM and in SANE, appears relevant in ECOB. On the other hand, differently from ECOB, FOM allows for an immediate computation, whereas EC for a very long-term analysis of fragmentations (e.g. more than 100 years). 


\subsection{Comparison with CONCEPT}

The tool developed by the University of Southampton, independently from this work, CONCEPT, is based on the capabilities of the evolutionary model, DAMAGE, and a two-stage process to evaluate the criticality of a given object. Firstly, the evolution of the background population is simulated with DAMAGE and its state at different time steps is saved and stored. Secondly, the fragmentation of the object is superimposed to the scenario, by propagating all fragments produced by the breakup and larger than $10 \mathrm{~cm}$. At each time step CONCEPT evaluates the probability of a collision for the fragments, so that consequent events can be modelled. The criticality index $C_{\text {dam }}$ is based on the difference in the total number of objects between the case with fragmentation and the reference case with no breakup. A Monte Carlo approach is adopted to give statistical meaning to the results. An alternative criticality index $P_{\text {con }}$ is defined; it indicates the probability that a run with the fragmentation has a larger number of objects than the reference scenario (Lewis, 2015).

Table 6 presents a subset of the objects analysed by Lewis (2015): only objects in LEO in orbits between 700 and $1000 \mathrm{~km}$ were analysed. Table 6] shows for each object its main features and the criticality as computed in DAMAGE, CONCEPT, and with the proposed index ECOB.

Analysing the results more in detail, Figure 23 shows the correlation between the criticality index computed by DAMAGE and ECOB. The two methods appear to give different results, especially for the three objects (ID 4, 7, 12) highlighted in the figure. These three objects have relative high altitude that results in a long orbital lifetime of their fragments. The simulation time frame is equal to 200 years versus only 25 years for ECOB, so this may explain the difference in the results as 25 years may not be enough to observe the large effect of the altitude. However, this does not explain why the index $C$ for ID 1 and ID 8 is not as high as for ID 4 .

Figure 24 shows the comparison with the criticality index computed in CONCEPT. Also in this case there is not a strong correlation between the indices. The large difference in the evaluation of the object with ID 12 is due to the 


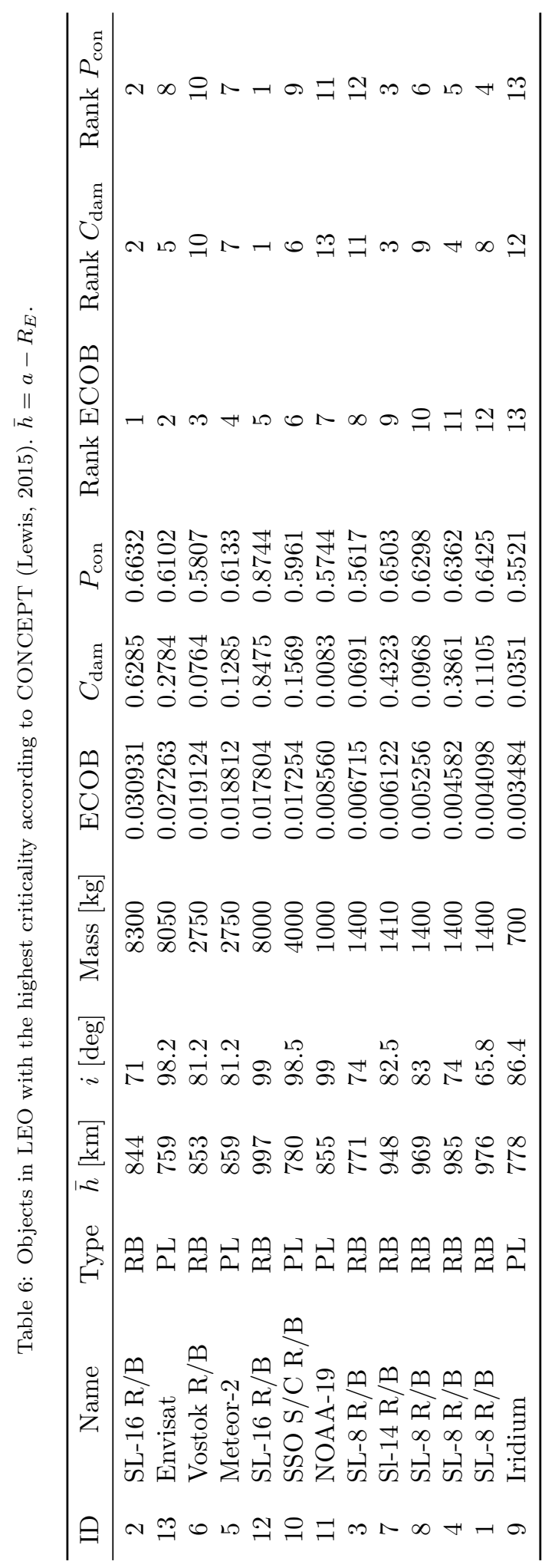




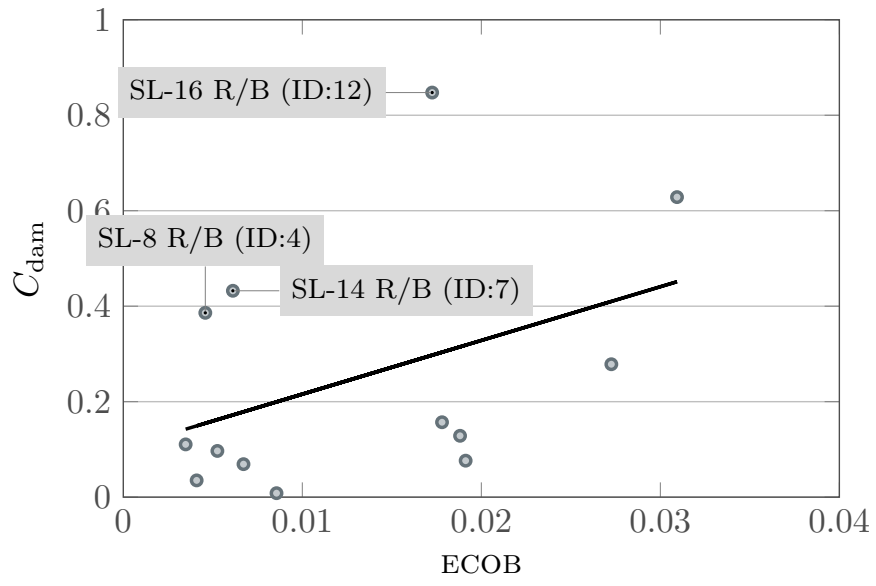

Figure 23: Correlation between the proposed index ECOB and the criticality index computed in DAMAGE.

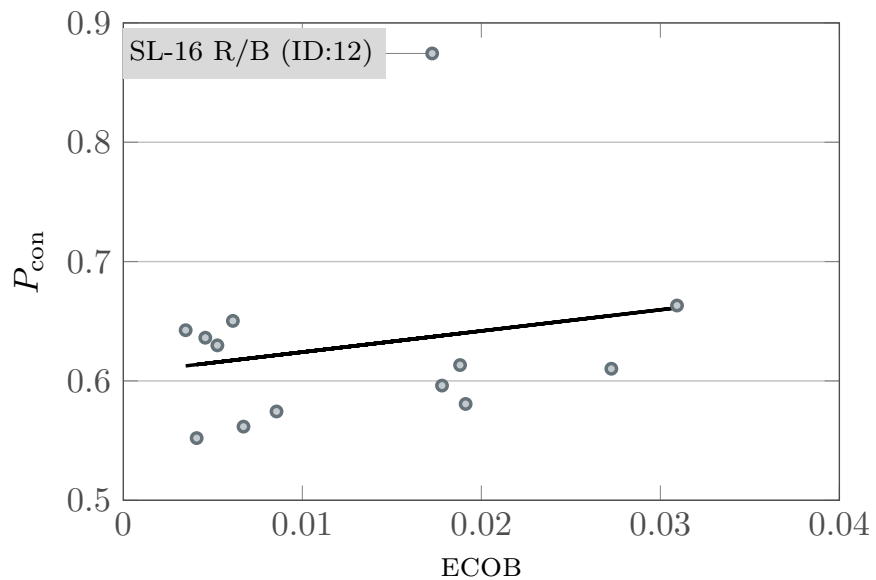

Figure 24: Correlation between the proposed index ECOB and the criticality index computed in CONCEPT. 
fact that this object generates a large feedback effect when CONCEPT is used.

This object has a large mass, so it produces a large cloud with fragments with long orbital lifetime due to the high initial altitude. For this reason, the fragments can collide with other objects and generate new fragmentations. This behaviour was partially present also in the simulation with DAMAGE and it is the reason why this object has a such a large criticality index according to the analysis by Lewis (2015). In the proposed index ECOB the feedback effect cannot be modelled, so this can explain the difference in the results.

\subsection{Comparison with CSI}

Rossi et al. (2015b) define a criticality index that takes into account four key-elements: environmental dependence, lifetime dependence, mass, and inclination. All these factors are combined in one index, $\Xi$, that is called the Criticality of Spacecraft Index. The expression of $\Xi$ is

$$
\Xi=\frac{M}{M_{0}} \frac{D(h)}{D_{0}} \frac{\operatorname{life}(h)}{\operatorname{life}\left(h_{1000}\right)} \frac{1+k \Gamma(i)}{1+k},
$$

where

- $\frac{M}{M_{0}}$ factors in the mass of the analysed spacecraft $M$, divided by a reference mass $M_{0}=10000 \mathrm{~kg}$

- $\frac{D(h)}{D_{0}}$ considers the effect of the environment through $D(h)$, which is the spatial density of objects at the orbital altitude $h$, normalised by $D_{0}$, the spatial density of objects at $770 \mathrm{~km}$

- $\frac{\text { life }(h)}{\operatorname{life}\left(h_{1000}\right)}$ compares the expected orbital lifetime of the object given its orbital altitude $h$ to the orbital lifetime of an object with $h_{1000}=1000 \mathrm{~km}$

- $\frac{1+k \Gamma(i)}{1+k}$, with $k=0.6$ and $\Gamma(i)=(1-\cos i) / 2$, considers the effect of inclination.

The objects evaluated by Rossi et al. (2015b) and their value of $\Xi$ are listed in Table 7, where also the value of ECOB is reported. Figure 25 shows clearly 


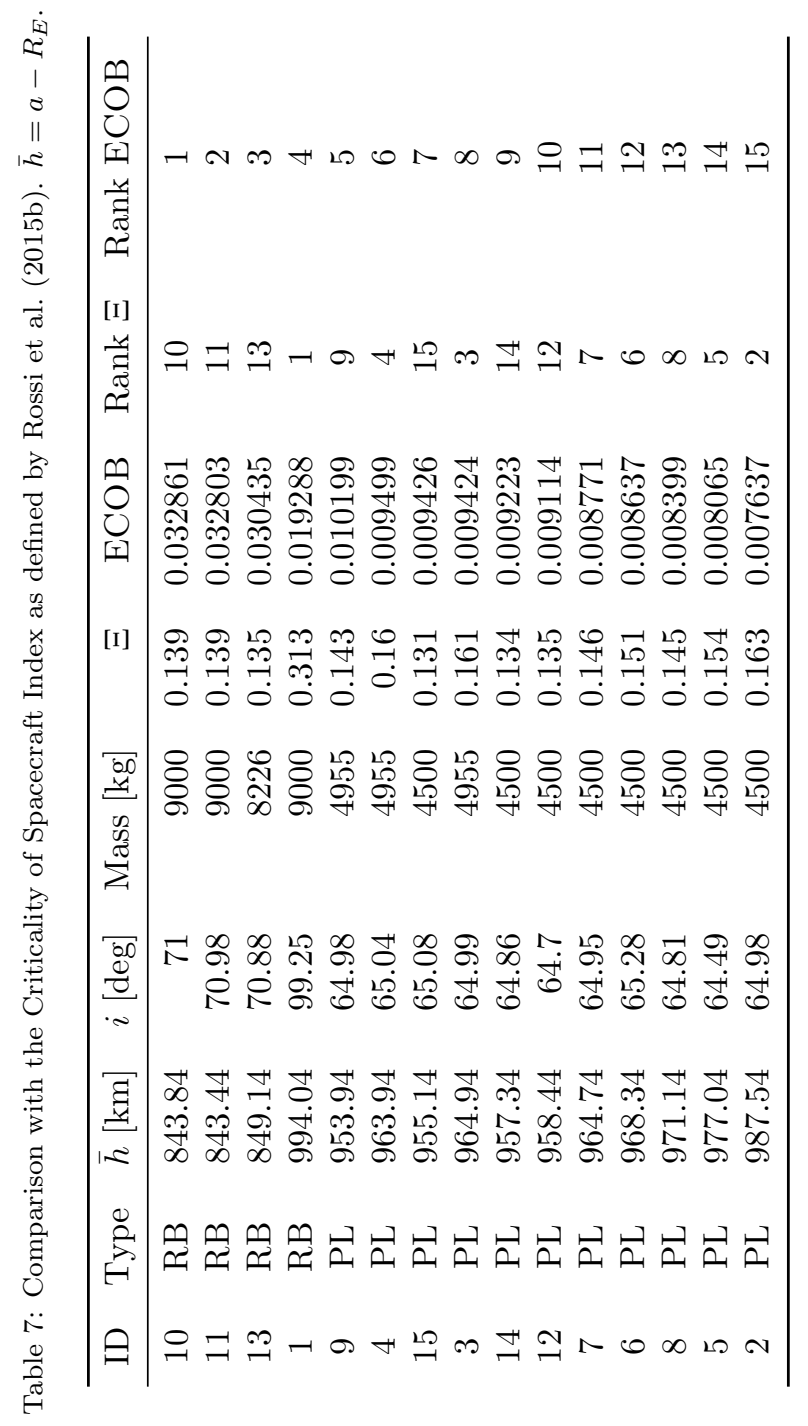


that the two indices predict very different criticality for the objects in Table 7 As in the case of CONCEPT, the same kind of object (a SL-16 R/B at high altitude) presents a much higher criticality index than the others. The simple expression of $\Xi$ allows to analyse in detail the reason of this result. Figure 26 compares the four components of $\Xi$ for all the tested objects. The spacecraft with ID1 presents the largest mass, the longest lifetime, and the most critical inclination among all 15 objects. When ECOB is computed the mass and the inclination contribute to a large value of the criticality index, but the altitude actually reduces the criticality of the object. In fact, its orbit appears to be far from the ones with the largest effect on the selected targets (Figure 27). The opposite happens for the objects with ID 10, 11, 13, which have a large ECOB because they are in an orbital region with a large influence on the reference targets, but a small $\Xi$ because of their orbital lifetime. The same observation explains the negative correlation in the cluster of object: 5 in the bottom left of Figure 25. for these objects, ECOB increases when their altitude decreases because their distance from the targets' orbits decreases, whereas $\Xi$ increases if the altitude increases. This observation reflects the fact that two indices are measuring the environmental impact on two distinct set of objects: ECOB only active satellites in a medium term timespan, whereas $\Xi$ on the whole LEO region with an indefinite timespan. Both descriptions are possible, but it should be clarified which is more relevant to rank the criticality of space objects.

\section{Conclusions}

This paper proposed an environmental index based on the assessment of the effect of breakups on operational satellites. The population of operational satellites is analysed by considering the distribution of the spacecraft cross-sectional area in semi-major axis and inclination, for satellites in orbit between 700 and

\footnotetext{
${ }^{5}$ These objects have orbital parameters similar to Cosmos satellites launched in 1976, 1986, 1988; however, the mass in Table 7 does not match the data in DISCOS database, so it was not possible to identify the objects.
} 


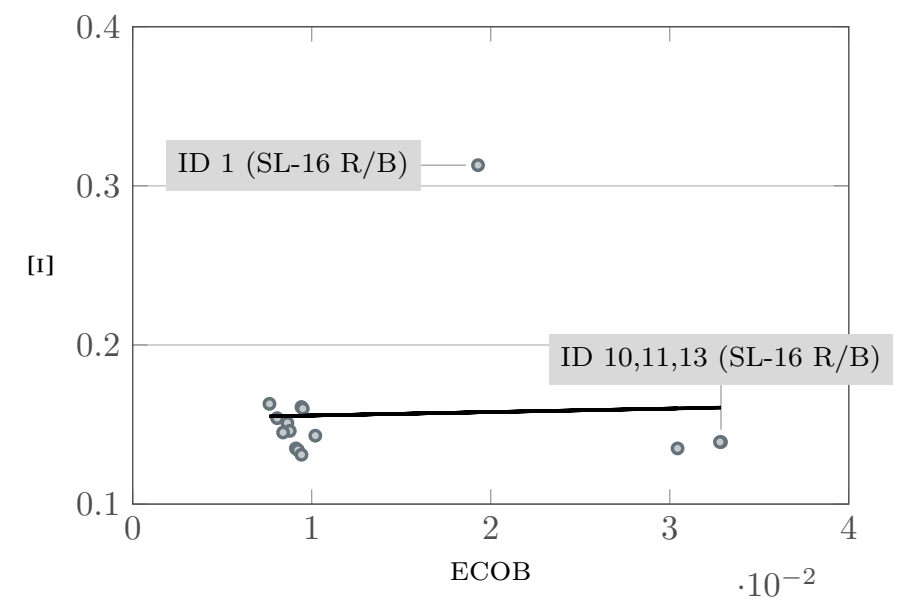

Figure 25: Correlation between the proposed index ECOB and $\Xi$ as defined by Rossi et al. (2015b).

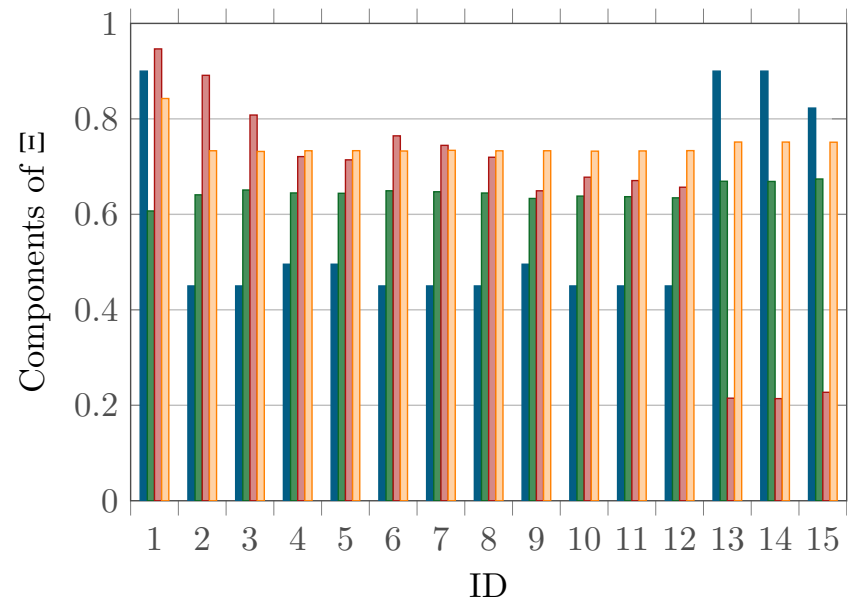

IIMass $\square \square$ Environment $\square \square$ Lifetime $\square \square$ Inclination

Figure 26: Analysis of the components of $\Xi$ for the objects in Table 7 


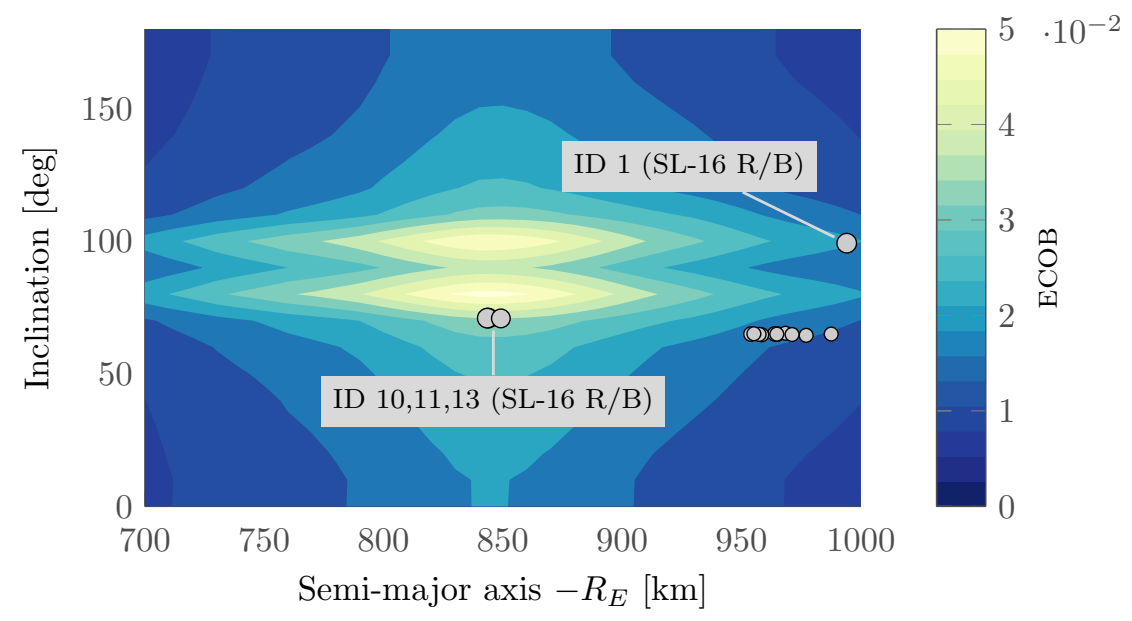

Figure 27: Contour plot of the environmental index ECOB for a breakup mass equal to $10000 \mathrm{~kg}$ and objects from Table 7 The size of the marker is proportional to the mass of the object.

$1000 \mathrm{~km}$. The distribution was sampled in such a way to obtain a set of representative targets, whose trajectories are propagated and which are used to compute the collision probability due to the simulated fragmentation.

For the fragmentation initial conditions, a variation in semi-major axis, inclination, and mass is considered. The simulated breakup is a catastrophic collision so that, applying the NASA breakup model, a direct dependence of the number of produced fragments on the fragmenting mass can be included. Once the debris cloud is generated, its density is propagated by applying the analytical method CiELo to describe the effect of atmospheric drag. Given the spatial density of the cloud, the collision probability of the targets is obtained by applying the kinetic theory of gases. The sum of the cumulative collision probability over 25 years for all the representative targets gives the value of the environmental index.

The index is computed for some defined grid points in semi-major axis and inclination, whereas the values for different masses can be derived by simply rescaling the results corresponding to a reference mass value. The environmental index for a generic space object is obtained by interpolation of the values 
on the grid points. The environmental index was assessed for all the objects present in the DISCOS database and in orbit between 700 and $1000 \mathrm{~km}$. The results appear consistent and highlight the contribution of the inclination, with maximum values of the environmental index for the fragmentations occurring at orbital inclinations close to the targets' one. In this configuration, the targets cross the fragment cloud at the latitudes with maximum density, resulting in high collision probability. Also the fragmentation altitudes affects the environmental index, which is higher for altitudes close to the ones populated by operational spacecraft. The proposed environmental index was compared to other four formulations proposed in the literature, which consider different aspects of the problem (e.g. distinguishing between active and inactive objects, evaluating the likelihood of the fragmentation to happen). For all cases, an explanation of the differences in the results was proposed. The comparison also suggests the importance of a clear definition of the scope and purpose of environmental indices to identify which aspects are essential to their definition.

\section{Acknowledgements}

Francesca Letizia acknowledges the support from the Institution of Engineering and Technology to organise a visiting period at ESA Space Debris Office. The authors thank Jan Siminski and Stijn Lemmens, from the ESA Space Debris Office, for their support, and Dr. Sergio Ventura, from ESA Independent Safety Office, for his precious feedback on the index formulation. The authors acknowledge the use of the IRIDIS High Performance Computing Facility, and associated support services at the University of Southampton, in the completion

of this work. Part of this work was funded by EPSRC DTP/CDT through the grant EP/K503150/1.

\section{References}

Bastida Virgili, B., Krag, H., 2013. Active Debris Removal for Leo Missions, in: Ouwehand, L. (Ed.), Sixth European Conference on Space Debris, ESA 
Communications. pp. 22-25.

Colombo, C., 2014. Semi-analytical propagation for highly elliptical orbits to build maps for end-of-life disposal, in: International workshop on Key Topics in Orbit Propagation Applied to Space Situational Awareness, Logroño.

European Cooperation for Space Standardisation, 2009. Space product assurance: Failure modes, effects (and criticality) analysis (FMEA/FMECA). Technical Report ECSS-Q-ST-30-02C. ESARequirementsandStandardsDivision.

Flohrer, T., Lemmens, S., Bastida Virgili, B., Krag, H., Klinkrad, H., Parrilla, E., Sanchez, N., Oliveira, J., Pina, F., 2013. DISCOS - Current status and future developments, in: Ouwehand, L. (Ed.), Sixth European Conference on Space Debris, ESA Communications.

Jenkin, A., 1996. Probability of collision during the early evolution of debris clouds. Acta Astronautica 38, 525-538. URL: http: //wWw.sciencedirect.com/science/article/pii/0094576596000598, doi:10.1016/0094-5765(96)00059-8.

Johnson, N.L., Krisko, P.H., 2001. NASA's new breakup model of EVOLVE 4.0. Advances in Space Research 28, 1377-1384. URL: http://www. sciencedirect.com/science/article/pii/S0273117701004239, doi 10 . 1016/S0273-1177(01)00423-9.

Kebschull, C., Krag, H., Braun, V., Hesselbach, S., Radtke, J., Scheidemann, P., 2014a. Simulation of the space debris environment in leo using an analytical approach, in: 40th COSPAR Scientific Assembly.

Kebschull, C., Radtke, J., Krag, H., 2014b. Deriving a priority list based on the environmental criticality, in: 65th International Astronautical Congress, International Astronautical Federation. IAC-14.A6.P48.

Kessler, D.J., 1981. Derivation of the collision probability between orbiting objects: the lifetimes of Jupiter's outer moons. Icarus 
48, 39-48. URL: http://linkinghub.elsevier.com/retrieve/pii/ 0019103581901512, doi $10.1016 / 0019-1035(81) 90151-2$.

King-Hele, D., 1987. Satellite orbits in an atmosphere: theory and application. Blackie, Glasgow and London.

Krisko, P.H., 2011. Proper Implementation of the 1998 NASA Breakup Model. Orbital Debris Quarterly News 15,1-10. URL: http://orbitaldebris.jsc. nasa.gov/newsletter/pdfs/ODQNv15i4.pdf.

Lang, T., Destefanis, R., Evans, L., Grassi, L., Kempf, S., Schaefer, F., Donath, T., 2013. Assessing Debris Mitigation Efficiency Using Risk- Oriented Criteria : Application To Leo European Mission, in: Ouwehand, L. (Ed.), Sixth European Conference on Space Debris, ESA Communications. pp. 22-25.

Letizia, F., 2016. Space debris cloud evolution in Low Earth Orbit. Ph.D. thesis. University of Southampton.

Letizia, F., Colombo, C., Lewis, H.G., 2015a. 2D continuity equation method for space debris cloud collision analysis, in: 25th AAS/AIAA Space Flight Mechanics Meeting, AAS/AIAA. AAS 15-293.

Letizia, F., Colombo, C., Lewis, H.G., 2015b. Analytical model for the propagation of small debris objects clouds after fragmentations. Journal of Guidance, Control, and Dynamics 38, 1478-1491. URL: http://arc.aiaa.org/doi/ abs/10.2514/1.G000695, doi:10.2514/1.G000695.

Letizia, F., Colombo, C., Lewis, H.G., 2015c. Collision probability due to space debris clouds through a continuum approach. Journal of Guidance, Control, and Dynamics URL: http://arc.aiaa.org/doi/abs/10.2514/1.G001382, doi:10.2514/1.G001382 accessed 10 September 2015 .

Letizia, F., Colombo, C., Lewis, H.G., 2015d. Multidimensional extension of the continuity equation method for debris clouds evolution. Advances in Space Research 57, 1624-1640. URL: http://www.sciencedirect.com/science/ article/pii/S0273117715008327, doi 10.1016/j.asr.2015.11.035 
Lewis, H.G., 2014. ACCORD: Alignment of Capability and Capacity for the Objective of Reducing Debris. FP7 Final Report. University of Southampton. URL: http://cordis.europa.eu/docs/results/262/ 262824/final1-accordfinalreportsection4-1.pdf

Lewis, H.G., 2015. Fragmentation Consequence Analysis for LEO and GEO Orbits. Technical note: Analytical Model. Technical Report ESA Contract No. 4000106543. University of Southampton.

McInnes, C.R., 1993. An analytical model for the catastrophic production of orbital debris. ESA Journal 17, 293-305.

McKnight, D.S., 1990. A phased approach to collision hazard analysis. Advances in Space Research 10, 385-388. URL: http: //www.sciencedirect.com/science/article/pii/0273117790903749, doi:10.1016/0273-1177(90)90374-9.

Orbital Debris Program Office, 2014. History of on-orbits satellite fragmentations. URL: http://orbitaldebris.jsc.nasa.gov/library/ satellitefraghistory/13theditionofbreakupbook.pdf. [Online] Retrieved on $26 / 08 / 2015$.

Radtke, J., Kebschull, C., Flegel, S., Braun, V., Schäfer, F., Rudolph, M., Utzmann, J., 2014. Fragmentation Consequence Analysis for LEO and GEO Orbits. Technical Report ESA Contract No. 4000106517/12/F/MOS. Technische Universität Braunschweig.

Rossi, A., Anselmo, L., Cordelli, A., Farinella, P., Pardini, C., 1998. Modelling the evolution of the space debris population. Planetary and Space Science 46, 1583-1596. URL: http://www.sciencedirect.com/science/article/ pii/S0032063398000701, doi:10.1016/S0032-0633(98)00070-1

Rossi, A., Lewis, H.G., White, A.E., Anselmo, L., Pardini, C., Krag, H., Bastida Virigili, B., 2015a. Analysis of the consequences of fragmentations in 
Low and Geostationary orbits. Advances in Space Research URL: http://dx. doi.org/10.1016/j.asr.2015.05.035, doi:10.1016/j.asr.2015.05.035.

Rossi, A., Valsecchi, G.B., Alessi, E.M., 2015b. The Criticality of Spacecraft Index. Advances in Space Research 56. URL: http://dx.doi.org/10.1016/ j.asr.2015.02.027, doi $10.1016 /$ j.asr.2015.02.027, special Issue: Advances in Asteroid and Space Debris Science and Technology - Part 1.

Su, S.Y., Kessler, D., 1985. Contribution of explosion and future collision fragments to the orbital debris environment. Advances in Space Research 5, 25-34. URL: http://dx.doi.org/10.1016/0273-1177(85)90384-9, doi 10.1016/ 0273-1177(85)90384-9.

Sykes, M., 1990. Zodiacal dust bands: Their relation to asteroid families. Icarus 9. URL: http://www.sciencedirect.com/science/article/pii/ $001910359090117 \mathrm{R}$.

Utzmann, J., Oswald, M., Stabroth, S., Voigt, P., Retat, I., 2012. Ranking and characterization of heavy debris for active removal, in: 63rd International Astronautical Congress, International Astronautical Federation. IAC-12-A6.2.8.

Valk, S., Lemaître, A., Deleflie, F., 2009. Semi-analytical theory of mean orbital motion for geosynchronous space debris under gravitational influence. Advances in Space Research 43, 1070-1082. URL: http://www. sciencedirect.com/science/article/pii/S0273117708006807, doi 10 . $1016 / j \cdot a s r .2008 .12 .015$ 